\title{
Chapter 41 \\ Cloud, Edge, and Mobile Computing for Smart Cities
}

\author{
Qian Liu, Juan Gu, Jingchao Yang, Yun Li, Dexuan Sha, Mengchao Xu, \\ Ishan Shams, Manzhu Yu, and Chaowei Yang
}

\begin{abstract}
Smart cities evolve rapidly along with the technical advances in wireless and sensor networks, information science, and human-computer interactions. Urban computing provides the processing power to enable the integration of such technologies to improve the living quality of urban citizens, including health care, urban planning, energy, and other aspects. This chapter uses different computing capabilities, such as cloud computing, mobile computing, and edge computing, to support smart cities using the urban heat island of the greater Washington DC area as an example. We discuss the benefits of leveraging cloud, mobile, and edge computing to address the challenges brought by the spatiotemporal dynamics of the urban heat island, including elevated emissions of air pollutants and greenhouse gases, compromised human health and comfort, and impaired water quality. Cloud computing
\end{abstract}

Q. Liu · J. Yang · Y. Li · D. Sha $\cdot$ M. Xu · I. Shams · M. Yu · C. Yang ( $\varangle)$

NSF Spatiotemporal Innovation Center \& Department of Geography and GeoInformation Science, George Mason University, Fairfax, USA

e-mail: cyang3@gmu.edu

Q. Liu

e-mail: qliu6@gmu.edu

J. Yang

e-mail: jyang43@gmu.edu

Y. Li

e-mail: yli38@gmu.edu

D. Sha

e-mail: dsha@gmu.edu

M. Xu

e-mail: mxu6@gmu.edu

I. Shams

e-mail: ishams@gmu.edu

M. Yu

e-mail:myu7@gmu.edu

J. Gu

Beijing Institute of Surveying and Mapping, Beijing, China

e-mail: gujuan@bism.cn 
brings scalability and on-demand computing capacity to urban system simulations for timely prediction. Mobile computing brings portability and social interactivity for citizens to report instantaneous information for better knowledge integration. Edge computing allows data produced by in-situ devices to be processed and analyzed at the edge of the network, reducing the data traffic to the central repository and processing engine (data center or cloud). Challenges and future directions are discussed for integrating the three computing technologies to achieve an overall better computing infrastructure supporting smart cities. The integration is discussed in aspects of bandwidth issue, network access optimization, service quality and convergence, and data integrity and security.

\subsection{Introduction}

\subsubsection{Why Computing is Important in Smart Cities}

Increasing global urbanization generates many problems, such as traffic congestion, energy consumption, industrial waste, and heat islands (Rao and Rao 2012; González-Gil et al. 2014; Li et al. 2012; Zhong et al. 2017; Rizwan et al. 2008). These problems produce serious negative impacts on urban residents. For example, an urban heat island (UHI) in an urban area or metropolitan area is significantly warmer than its surrounding rural areas due to human activities. UHI contributes directly to environmental warming, industrial waste, air pollution, and heat-related mortality (Petkova et al. 2016). In order to alleviate urban problems and achieve sustainable development, a number of smart-city solutions have been the subject of experiments in cities over the past two decades. Copenhagen Municipality uses monitor sensors installed in different trash containers and information systems to optimize waste handling (State of Green Denmark 2018). Seoul of South Korea has smart meters installed in residential houses, office areas, and industrial facilities to report in real time the consumption of electricity, water, and gas (Hwang and Choe 2013). Smart cities are supported by key information and communications technologies (ICT) including the Internet of things (IoT), computing platforms, big data, artificial intelligence (AI), geographical information, and others (Graham and Marvin 2002; Morán et al. 2016; Mitchell et al. 2013) (Fig. 41.1). Among them, diverse sensors, stable communication networks, and sophisticated computing platforms are three fundamental technologies for smart cities. Sensors are the smart-city's sensory organs, to capture and integrate data continuously in real time. Smart sensors, such as monitoring cameras, smart meters, and wearable devices, are widely employed to improve urban transportation, utility planning, parking-lot management, pollution monitoring, and health care. The number of connected devices on the Internet will exceed 50 billion by 2020 according to Cisco (2017). The communication network is the smart-city's transmission system, transmitting data from sensors to computing platforms. Reliable, scalable, and high-speed networks, including wired and wireless 


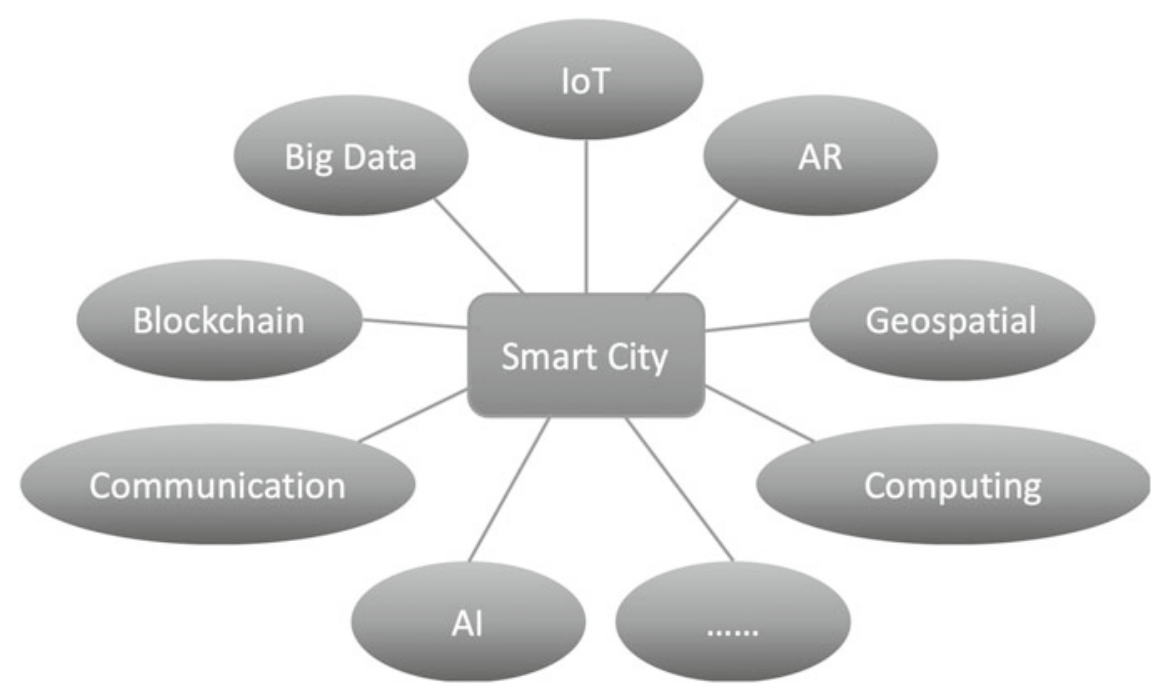

Fig. 41.1 Key technologies of smart cities

networks, are fundamental infrastructure for such transmission. Computing platforms support the management and analyses of relevant city data in a broader context, to identify city-relevant events that require processing and action. A large quantity of data is generated continuously from countless smart-city sensors. To store, process, and analyze the massive heterogeneous data, a stable, scalable, fast computing platform is required. For example, car drivers need a smart navigation system to provide them with the optimal driving route in real time, updated dynamically with traffic pattern and congestion changes. Different systems and devices using ICT have been developed to monitor and forecast UHI in the past years. For example, France developed a Heat Health Watch Warning System to monitor heat waves that may result in a large increase of mortality (Casanueva et al. 2019). Greece developed a UHI modeling system to simulate and forecast heat islands in Athens (Giannaros et al. 2014). Richmond has handmade devices equipped in cars and bikes to map UHI (Hoffiman 2018).

\subsubsection{Major Computing Techniques in Smart City Studies}

Washburn et al. (2009) described the smart city as using a collection of smart computing technologies to manage critical infrastructure components and services. A centralized cloud-computing architecture has been widely deployed in smart cities to extend the storage capability and improve the processing velocity with characteristics of elastically, on-demand, and pay-as-you-go computing resources (Yang and Huang 2013). Cloud computing maximizes the utilization rate of physical resources 
by adopting a series of technologies including virtualization and network security. Virtualization is a core technology supporting cloud computing, and abstracts actual hardware as virtual computer systems. Virtualization enables multiple operating systems to run on a computer system simultaneously and optimizes the use of computing and storage resources. Practically, cloud computing virtualizes computer resources and manages them in a resource pool to provide computing services over the network, reducing the idle time of resources including CPU, RAM, network, and storage. Public clouds (e.g., Amazon AWS, Microsoft Azure) are open to the public, who pay to use them. On the other hand, a private cloud is delivered via a secure private network and usually shared among people in a single organization. Cloud computing provides the smart city with the computing capability to store and access data and applications outside local computing environment through computer networks (Kakderi et al. 2016).

The proliferation of IoT enables smart cities to collect a large number of data and deploy a lot of applications at the edge to utilize these data (Shi et al. 2016). The data and applications also produce challenges of near-real-time response, privacy, and massive numbers of data for network transmission. Cloud computing alone is not sufficient to address such challenges. A new computing paradigm, edge computing, which shifts the data storage, processing and analyses to the end of the network, as close as possible to the devices, is deployed (Shi et al. 2016). With the aid of edge computing, the edges of network become data producers as well as data processors, addressing the challenge of response time, bandwidth, data safety, and privacy (Shi et al. 2016). Edge computing offers a number of benefits, including allowing services to continue to operate when there is no connection to the Internet, and processing data locally. This significantly reduces the network load with only processing results (which are normally smaller in volume than raw data) being transmitted across the network.

The past two decades have witnessed the increasingly use of mobile devices (such as mobile phones, portable computers, wearable devices, and smart vehicles) and rapid growth of wireless communication technology (Hashim Raza Bukhari et al. 2018). Data processing is shifted away from centralized computing centers to the mobile devices of end user. With battery volume and network bandwidth limitations, computing resources offered by mobile computing are not as reliable as the other two computing frameworks. Nevertheless, they are portable and able to collect and process data where cloud computing and edge computing are unavailable.

The three computing paradigms collaboratively provide a comprehensive and reliable data store and processing framework to overcome the disadvantages of a single device and enable a suite of applications of smart cities (Table 41.1) including: transport and traffic management, utilities and energy management, environmental protection and sustainability, public safety, and smart-city security.

Figure 41.2 illustrates the sensors and computing devices of a smart city and places them into three types: different sensors collecting different information for different purposes. The sensors also have embedded computing capabilities; for example, moving sensors can be used to provide flexible data collection to dynamically cover different regions with fast situation-aware processing capabilities such as navigation. 
Table 41.1 Application examples of cloud, edge and mobile computing in smart cities

\begin{tabular}{l|l|l|l}
\hline $\begin{array}{l}\text { Application } \\
\text { examples }\end{array}$ & \multicolumn{2}{|l}{ Computing paradigm } & Mobile computing \\
\cline { 2 - 4 } $\begin{array}{l}\text { Transport and } \\
\text { traffic } \\
\text { management }\end{array}$ & $\begin{array}{l}\text { Using cloud } \\
\text { computing for } \\
\text { smart-city logistics } \\
\text { (Nowicka 2014) }\end{array}$ & $\begin{array}{l}\text { Connected parking } \\
\text { meters (David 2018) }\end{array}$ & $\begin{array}{l}\text { Location-aware mobile } \\
\text { applications (Altman } \\
\text { et al. 2015) }\end{array}$ \\
\hline $\begin{array}{l}\text { Utilities and } \\
\text { energy } \\
\text { management }\end{array}$ & $\begin{array}{l}\text { Using cloud } \\
\text { computing for smart } \\
\text { grid energy } \\
\text { management (Bera } \\
\text { et al. 2015) }\end{array}$ & $\begin{array}{l}\text { Street lighting (David } \\
\text { 2018) }\end{array}$ & $\begin{array}{l}\text { The use of GPRS } \\
\text { technology for electricity } \\
\text { network telecontrol } \\
\text { (Souza et al. 2016) }\end{array}$ \\
\hline $\begin{array}{l}\text { Environmental } \\
\text { protection and } \\
\text { sustainability }\end{array}$ & $\begin{array}{l}\text { Using cloud } \\
\text { computing for climate } \\
\text { analysis and } \\
\text { simulation (Yang et al. } \\
\text { 2017a, b) }\end{array}$ & $\begin{array}{l}\text { Vehicular pollution } \\
\text { system based on IoT } \\
\text { Sushikesh and }\end{array}$ & $\begin{array}{l}\text { Location-aware weather } \\
\text { report applications } \\
\text { (Altman et al. 2015) }\end{array}$ \\
\hline $\begin{array}{l}\text { Public safety and } \\
\text { smart-city } \\
\text { security }\end{array}$ & $\begin{array}{l}\text { Cloud computing } \\
\text { services in medical } \\
\text { heath care solutions } \\
\text { (Kaushal and Khan } \\
\text { 2014) }\end{array}$ & $\begin{array}{l}\text { Smart home (Shi et al. } \\
\text { 2016) }\end{array}$ & $\begin{array}{l}\text { Healthcare applications } \\
\text { (Hameed 2003) } \\
\text { Lost child application } \\
\text { (Satyanarayanan 2010) }\end{array}$ \\
\hline
\end{tabular}

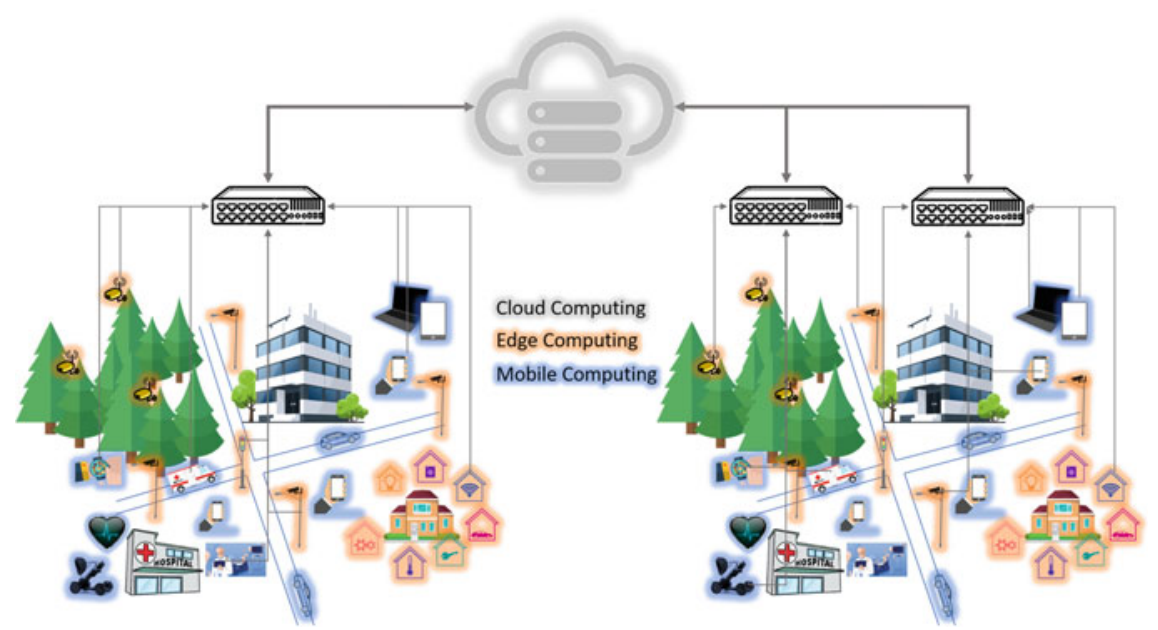

Fig. 41.2 Urban computing for smart cities include cloud computing (gray), edge computing (orange), and mobile computing (blue) devices and capabilities 
Edge-computing sensors act as fixed data collectors with various computing powers depending on tasks assigned; for example, a higher edge-computing capacity enables handling analytics for a larger area, like a neighborhood. All data and processes can also be uploaded to the cloud's centralized computing, for extensive data processing and knowledge extraction or mining.

Computing serves as an indivisible capability to support effective and efficient smart-city applications and research, through which massive smart-city data can be processed in parallel and in a real-time manner. This chapter introduces the three computing paradigms' engagement in a smart city using UHI as a case study. A workflow was proposed to integrate three computing techniques as a seamless integration for handling UHI problem (one of the severe urban challenges facing us today especially with climate and global change).

This chapter starts with an introduction to urban computing in 41.1, followed by the current status and challenges of computing in different smart-city scenarios. Sections. 41.3, 41.4 and 41.5 introduce, respectively, cloud computing, edge computing, and mobile computing using UHI as a use case. The last section uses UHI as an example to integrate the three computing paradigms through collaborative workflow.

\subsection{Computing for Smart Cities}

\subsubsection{Data and Model in Smart Cities}

Smart cities require multiple data sources and reliable models to produce decisionsupporting information. It becomes especially challenging when a massive number of smart devices and sensors are engaged. This section introduces five typical smartcity applications, the data engaged, corresponding models, and their requirements for computing.

\subsubsection{Transport and Traffic Management}

Transportation is one of the most important aspects for urban-living activities. Various sources of transportation data are related to people's travel and commuting, which is a complicated and indispensable part of smart cities. For example, traffic data are generated and collected by sensors in traffic vehicles (e.g., taxis, buses, metros, trains, vessels, and planes) or monitors installed along the roads (e.g., loop sensors and surveillance cameras). Commuting data refer to data that record people's regular movement in cities. Geo-tagged social network data collect posts (e.g., blogs, tweets) through social networks which are tagged with geoinformation. Road network data represent road segments and intersections, respectively. The transportation network is modeled as a directed graph which includes transit routes and stop facilities of 
buses and metro networks. Point of interest (POI) data depict related information for facilities, such as restaurants, shopping malls, parks, airports, schools, and hospitals in the city, which helps guiding people to find their destinations.

To handle and integrate the complex data from different sources efficiently and to satisfy various user groups, different models are used for intelligent transportation systems, such as agent-based traffic management models (Sciences et al. 2011), cognitive rationality-based decision-making models (Cascetta et al. 2015) and mixedranked logit models (Liu et al. 2017).

\subsubsection{Utilities and Energy Management}

The large volume of data for utilities and energy management is increasingly adding burden to urban computing systems, especially with the wide adoption of sensors, wireless transmission, and network communication (Zhou et al. 2017). The input data of smart-city energy systems include numeric data, text-based data, and audio-visual data. Numeric data refer to the observations and collections from sensors and meters, such as power quality, customer usage, and electrical production. Text-based data sources are mainly internal and external communications, regulatory documents, legal documents, and linguistic social media records. Audio-visual data are records and social media data in the form of sound and video (Schuelke-Leech et al. 2015).

The utilities and energy management systems should be green, sustainable, and with high operational speed and efficiency. Schuelke-Leech et al. (2015) demonstrate how future sustainable energy systems will be smart and integrated with smart grids, renewable sources, storage, and energy management and monitoring systems. The energy and utility systems of cities are complicated because they have to satisfy a huge number of requirements with comparably limited supply. The computational systems need not only to integrate intermittent power sources efficiently and effectively, but also to predict equipment failures and power outages, allowing utilities to optimize their maintenance budgets. For example, Sheikhi et al. (2015) presented an Energy Hub Model in a future vision of energy systems, which supported real-time and two-way computational communication between utility companies and smart energy hubs. Such models also allowed intelligent infrastructures at both ends, since to manage power consumption necessitates large-scale real-time computing capabilities to handle the communication and the storage of big data. These systems help managers, employees, and consumers to make informed decisions based on data and empirical investigation, rather than on intuition or past practice.

\subsubsection{Environmental Protection and Sustainability}

Environmental protection and sustainability also play important roles in smart cities. The environmental resources refer to minerals, forests and grasslands, wetlands, rivers, lakes, and the ocean. These natural resources have been exploited unduly, and the inappropriate management of natural resources has caused severe environmental 
degradation (Song et al. 2017). The data that urban environmental protection and sustainability management systems are dealing with include hydrogeological data, environmental surveillance data, ecological statistics, and meteorological data. The data quantity and dimensions are big according to the characteristics of big data. The functions of these data are not only to accurately present the current situation of the environment but also to effectively predict the future and sustainability. Therefore, powerful computational ability is needed to help governments and individual users to prevent and settle environmental challenges.

As environmental protection and sustainability are important factors for the development of smart cities, data collection and computational models have flourished in this domain. Take the IoT and its associated computing model as an example: the informational landscape of smart sustainable cities and big data applications is augmented to achieve the required level of environmental sustainability (Bibri 2018). For governments, the combination of 3D GIS and cloud computing is also offering effective services in the environmental management of smart cities (Lv et al. 2018).

\subsubsection{Public Safety and Security}

Public safety and security are directly related to citizens' wellbeing and their lives. With the growth of different kinds of monitoring devices and systems, data from the IoT, unmanned aerial vehicles (UAV) (Menouar et al. 2017), and social media are leveraged to make our cities more and more safe and stable. Usually, the safety and security issues are directly related to people's life and property, and needs immediate and accurate response from relevant personnel. Therefore, extremely high performance in efficiency and accuracy is needed for safety and security models and systems. Edge and mobile computing, which can share the burden of the central cloud and improve processing speed, are ideal for the applications such as finding a lost child (Shi et al. 2016). Wearable devices and medical sensors can measure users' health conditions and send health data to the processing unit for doctors' further diagnosis.

To address these challenges, safety systems should include the following data sources and model features: health care and monitoring systems; smart safety systems for surveillance; smart systems of crisis management to support decision making, early warning, monitoring and forecasting emergencies; centrally operated units of police and integrated rescue systems (IRS); safe Internet connection and data protection; and centers of data processing (Lacinák and Ristvej 2017).

\subsubsection{Urban Heat Island and Urban Computing}

Urban computing utilizes the three computing paradigms to store, process, integrate, model, and analyze various big data and phenomena, such as real-time data generated by diverse smart sensors and devices, fundamental urban geographical data, social media data, data on transportation on flooding, and on UHI. UHI is considered one 
of the major urban challenges and is caused by a set of complex factors, including urban land use changes, solar radiation, anthropogenic heat sources, climate change, urban development, and wind speed and direction (Memon et al. 2009). The negative effects of UHI include: (1) increasing temperature in cities (Voogt and Oke 2003); (2) contribution to global warming (Van Weverberg et al. 2008; EPA 2016); (3) air pollution (Sarrat et al 2006; Davies et al. 2008); (4) increasing energy demand (Santamouris et al. 2001; Santamouris 2015); and (5) heat-related mortality (Guest et al. 1999; Conti et al. 2005; Haines et al. 2006; Filleul et al. 2006; Hondula, et al. 2014).

To reduce the negative impact of UHI, remotely sensed data, stationary meteorological monitoring data, building data, digital elevation data and other data were integrated to model, monitor, simulate, and evaluate UHI in more than 100 cities in the past 50 years. However, UHI studies involve big data storage, processing, and modeling, which need complicated computing. There is no single efficient computing architecture for large-scale or long-term UHI studies. This chapter takes UHI as an example to introduce how the combination of cloud, edge, and mobile computing can help addressing the smart city challenges in sequence of: (1) what are the computing challenges of smart cities; (2) how the three computing paradigms can help address the challenges; and (3) how to integrate the three computing paradigms to address these challenges using UHI as an example.

\subsubsection{Computing Challenges in Smart Cities}

\subsubsection{Big Data Handling}

Urban data have been harvested from various sources including (1) remote sensing, (2) in-situ sensing, (3) social sensing, (4) IoT sensing, and (5) simulation. The collected data together provide a comprehensive view of the urban system: for example, the underground water distribution network for water usage management (Karwot et al. 2016), real-time parking prediction (Vlahogianni et al. 2016), and 3D city modeling for urban disaster management (Amirebrahimi et al. 2016). However, the sensing and simulation produce large numbers of data that far exceed the storage capacity of an individual computer. Taking remote sensing as an example, fine spatiotemporal resolution imagery grows exponentially with spatial resolution. For example, the volume of the Earth Observing System and Data Information System (EOSDIS) data archive was more than 27.5 petabytes (PB) at the end of fiscal year 2018 (NASA Earth Science Data Systems Program Highlights 2018). Efficiently storing such a large volume of data is a challenging task. Meanwhile, data are produced in high velocity in a continuous manner with the development of advanced techniques, such as water meters, which collect water usage data in a fixed interval (e.g., every $30 \mathrm{~s}$ ). The velocity of data requires streaming data collection and analysis methods for near-real-time applications. In addition, the heterogeneous data are stored in various file formats, such as image, video, text, or audio, and pose grand challenges to data management. 


\subsubsection{Compute-Intensive Modeling and Processing}

The smart city is becoming a sophisticated ecosystem where massive data are being collected and innovative solutions are being proposed to deliver smart services (Anthopoulos 2015). Generally, those solutions rely on complicated data models and analytics with the aid of the computer. Data models often represent objects or situations in the real world, and a digital model makes mathematical analysis possible. For example, a trend in smart cities is to build three-dimensional (3D) models for visualization and analytics such as skyline analysis, underground utility management, and route selection (Yao et al. 2017; and see Sects. 41.5 and 41.6). Although a 3D model can represent cities as virtual reality to support real 3D analysis, more computing resources are needed for effective 3D rendering and analysis. Data analytics is an important component of the big data paradigm. However, it comes after data collection, deduplication, completion, aggregation, harmonization, contextualization, and filtering. These components of the process are essential to enable analytics to derive useful insights. Different types of computing resources are required for different components in the data process workflow. For example, moving partial computing resources to the data collection sites for data cleaning can reduce the volume of data transferred to the core computing platform, result in a lower bandwidth cost and a higher analysis speed.

\subsubsection{Data Security and Privacy}

Security and privacy issues are two of the major challenges in smart-city computing due to the identification information within the data and the security issues located in the multiple computing layers. Generally, some of the raw data may contain confidential or sensitive information related to people or governments; such data processing should be protected against unauthorized usage. Taking cellular data for example, a phone number in each record represents a real person and makes an individual's daily activities traceable, which may divulge the private affairs of people. In the water distribution management system, a methodology for synthetic household water consumption was proposed to reproduce water consumption data due to privacy constraints (Kofinas et al. 2018). Simultaneously, in smart-city applications, data move over various computing layers through networks, some of which may be insecure. In an application, data may be processed with more than one computing technique including edge computing, mobile computing, and cloud computing. In most cases, mobile devices and edge computing nodes need to connect via Wi-Fi to upload data to the cloud-computing platform. Connection to unauthorized Wi-Fi may bring security risks to the system. Besides network connection, distributed opensource big data platforms like Hadoop and Elastic search are becoming increasingly popular for distributed data storage and analytics, However, compared to commercial solutions, these platforms lack sufficient security guarantees (Sharma and Navdeti 2014). 


\subsubsection{Efficiency}

A trend in smart-city's applications is to extract information from big data, and thus, lack of efficiency becomes a bottleneck of most data-analytical applications. Different applications vary in levels of complexity and require different response times. Navigation needs immediate optimal route suggestion (e.g., fastest route option) based on real-time traffic data (Liebig et al. 2017). Predictions of hurricane intensity help people prepare for severe weather, saving properties, and human lives (Li et al. 2017). Applications like environmental sustainability are less sensitive to the response time. Meanwhile, although a series of open-source big data platforms, such as Apache Hadoop, Spark, HDFS, and MapReduce, have been developed and adopted in various domains, these platforms are not specifically designed to support spatiotemporal data. Performance issues are unavoidable when using these platforms to process spatiotemporal data without any modification. Some research has been done to customize these tools for domain adoption. Taking array-based raster data for example, a hierarchical index was proposed to speed up the query process of grid data stored in the HDFS file system (Hu et al. 2018). The development of an efficient spatiotemporal computing platform is still in an initial stage; how to utilize and optimize big data computing platforms to implement efficient smart-city applications remains a challenge.

\subsubsection{Generic Computing Architecture for Smart Cities}

Cloud, edge, and mobile computing support different functions and applications in the development of smart cities. To optimize the computation capability and further overcome the challenges discussed in Sect. 41.3, different types of computing paradigms should be utilized. Based on the characteristics and advantages of each type of computing, a computing architecture for a smart-city system is proposed (Fig. 41.3).

\subsubsection{General Computing Modules in Smart Cities}

The proposed architecture of computing system in smart cities contains the following five parts:

(1) Application acquisition: The function of the application layer is to collect requirements from users, then organize, and analyze them into the four aspects as mentioned in Sect. 41.2.1: Transportation and traffic management, utilities and energy management, environmental protection and sustainability, and public and smart-city security.

(2) Visualization: The visualization layer is designed to visualize the applications in the form of 2D and 3D maps, trajectories, images, charts, histograms, and others 

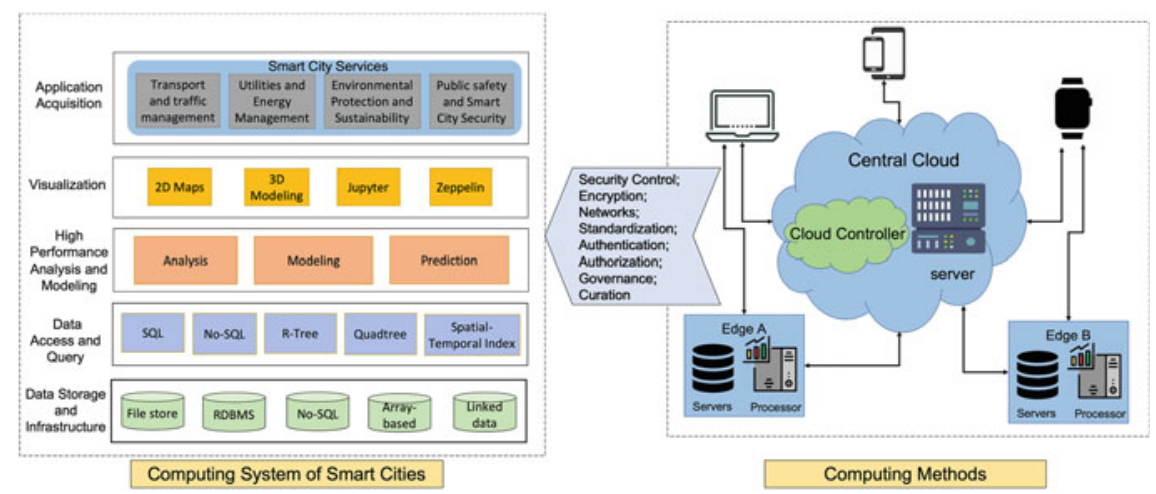

Fig. 41.3 Generic computing architecture for smart cities

using technologies and software such as 2D mapping, 3D modeling, Jupyter, and Zeppelin.

(3) High-performance analysis and modeling: As discussed in the former sections, computing for smart cities is usually encountered with big data issues, and high-performance computing techniques are essential to maintain a stable and efficient computation system. This layer implements data analysis, modeling, and prediction according to the applications.

(4) Data access and query: The system utilizes a data access and query layer to retrieve and select data sources that satisfy the needs and orders from users. Methods and techniques such as SQL, No-SQL, R-Tree, Quadtree, and spatialtemporal indexing will be adopted according to the category of data.

(5) Data storage and infrastructure: This layer provides the hardware and physical devices, including data storage facilities, as well as the servers and networks. The smart-cities-related data sources will be stored in different categories according to the requirements from uses, using database systems such as file storage, Relational Database Management System (RDMS), No-SQL, array-based, and linked-data databases.

\subsubsection{Computing Methods Integration}

Computing procedures are embedded in all the layers of the proposed computing architecture for smart cities, through a series of security controls, encryption, standardization, authentication, authorization, governance, curation, and network techniques. The core computing methods of smart cities contain central cloud computing, edge computing, and mobile computing. In the central cloud platform, data centers provide complex analysis and visualization capabilities, as well as hardware facilities and infrastructure for the cloud. The servers are linked with high-speed networks to provide services for clients. Normally, data centers are built and located in less populated places, with a high power-supply stability and a low risk of disaster (Dinh 
et al. 2013). The edge-computing platform is connected with the central cloud by the Internet. They have dual communication with each other to enable data interactions. The edge servers can share and reduce the burden of central servers, and as a result increase the speed of processing and delivering data. The mobile-computing platform is the mobile devices of the end users, which has a certain capability to process data along with mobility. Mobile devices can also be connected to central clouds by wireless networks for data transmission. Edge- and mobile-computing platforms are connected with each other in applications where interactions are needed.

In the architecture, the three computing paradigms are connected and assist each other, where there are distinctions between them in the collaboration of processing smart cities' services and applications. Different from cloud computing requiring all parts to be connected to the central cloud, where large volumes of data are processed to find optimization solutions or support decisions, edge computing relocates crucial data processing to the edge of the network, rather than constantly delivering data back to a central server. Therefore, edge-enabled devices can gather and process data in real time, allowing them to respond faster and more effectively, while mobile computing relates to the emergence of new devices and interfaces and has the data processing capability on the mobile devices. Moreover, the centralized cloud could perform extremely complex data processing, storing, and analytics. Edge computing usually performs less intricate data processing than central clouds, storing and forwarding. However, some mobile devices can only implement simple and limited data processing. By integrating the three computing paradigms, the efficiency challenges of intensive big data processing and computing can be remitted. Direct connection between edges, mobile devices, and the central cloud with a stable and secure network will guarantee the safety and security of the whole system.

\subsection{Cloud Computing for Smart Cities}

\subsubsection{Methodology}

Cloud computing is developed and improved based on the evolution of parallel computing, distributed computing, and grid computing (Jadeja and Modi 2012; Yang and Raskin 2009). Parallel computing allows many computation processes to run simultaneously, which achieves high performance in a divide-and-conquer fashion (Fu et al. 2015). Distributed computing contains components located on different networked computers which communicate and cooperate with each other to achieve a common computing objective (Yang et al. 2008). The inexpensive computer nodes and high-speed networks make possible the function of distributed computing systems (Jonas et al. 2017). Grid computing organizes a network of heterogeneous computer resources to work together and achieves high performance for processing and executing resource-hungry tasks like those normally allocated to supercomputers (Wang et al. 2018). Different from the above-mentioned computing modes, cloud 
computing is a model for enabling convenient, on-demand network access to a shared pool of configurable computing resources (NASA 2010), instead of a local machine or remote server handling applications.

Cloud computing is capable of scheduling and balancing the distribution of resources according to real utilization demand, and billing according to the usage. Using different techniques and according to different budgets, cloud computing extends subscription-based access to data, platforms, infrastructure, and software, approaches that are referred to as data as a service (DaaS), platform as a service (PaaS), infrastructure as a service (IaaS), and software as a service (SaaS) (Subashini and Kavitha 2011; Yang et al. 2011).

\subsubsection{Challenges, Motivations and Opportunities}

Past research (Gong et al. 2010; Zhang et al. 2010; Yang and Huang 2013; Mahmood 2011) identified the features and advantages of cloud computing as:

(1) Hyperscale. Some Internet companies have developed large-scale cloudcomputing platforms for business applications, and the practical clouds have a considerable scale. For example, Google cloud computing (Xiong et al. 2017) has millions of servers; Amazon, IBM, Microsoft, Saleforce, Ali, and Tencent (Hashem et al 2015; Rittinghouse and Ransome 2016), and other agencies have hundreds of thousands of servers in their clouds. Conceptually, a cloud can provide users with unprecedented computing power.

(2) Virtualization. Cloud-computing supports users to access services at any location using a variety of terminals and devices. The requested resources come from the cloud, which uses virtualization techniques to separate computer resources and services from underlying fixed physical entities (Gong et al. 2010). The application runs above in the cloud without specifying a server. Simple network connection enables users to benefit from super-powerful services via multiple devices, such as a computer, a PAD, or a mobile phone.

(3) Reliability. Cloud computing uses the capability of fault tolerance and isomorphic interchangeability of computing nodes and other strategies to ensure high reliability and availability (Dai et al. 2009). Compared with traditional in-house computing infrastructures, cloud computing is more reliable and consistent.

(4) Universality. Cloud computing is not specific to any particular applications. It can support a variety of applications under the support of a single cloud. The same cloud infrastructure can be shared by different applications at the same time (Yang et al. 2016).

(5) Scalability. The capabilities and scales of the cloud can be modified and extended dynamically to meet the needs of applications and growth (Lehrig et al. 2015). Scalability allows cost-effective running of workloads that make a very high demand on servers but only for short periods of time or occasionally. 
(6) On-demand. Users could request and receive access to cloud service offerings, like the traditional infrastructure utilities of water, electricity, and gas. Based on a pool of physical and virtual resources in the cloud, operations such as creating, stopping, and terminating could be conducted at any time without waiting for delivery and purchasing processes (Etro 2015). Usage monitoring tools of the cloud can record usage details for billing.

(7) Cost savings. The "pay-as-you-go" characteristic of cloud service enables personal and business clients to access the cloud from extremely cheap and price-flexible computing nodes. The automatic system of the cloud reduces the cost of data center management by deleting the basic maintenance budget. The lack of physical infrastructure removes the operational expenses of power, storage, administration and even labor costs.

Considering the advantages listed above, cloud computing can help to address the following computing challenges of smart cities:

(1) Unity and efficiency. Through the architecture of the IaaS model, cloud computing integrates various frameworks, hardware brands, and computing models of servers to the traditional data centers and provides a unified platform of application based on the cloud operating systems (Mitton et al. 2012). Meanwhile, with the virtualization techniques, cloud computing can be flexibly and effectively partitioned, allocated, and integrated over a potentially infinite number of storage and computing resources, and optimize the efficiency ratio according to application and requirements.

(2) Large-scale infrastructure. Infrastructure management of hardware and software is mainly responsible for the monitoring and management of large-scale foundational computing resources (Jin et al. 2014). Fundamental software resources include stand-alone operating systems, middleware, databases, and so on. Fundamental hardware resources include three main devices in the network environment: computing (server), storage (storage device), and network (switch, router, and other devices). The advantages of infrastructure management center are: (1) to manage the assets of the basic software and hardware resources; (2) to support the status and performance monitoring of the basic hardware; (3) to trigger alarms for abnormal situations, and remind users to maintain the abnormal equipment; (4) to carry out long-term statistical analysis of the basic software and hardware resources; and (5) to provide a decision-making basis for high-level resource scheduling.

(3) Sustainable and green energy. Facing the burden of large-scale fundamental software and hardware resources, green and energy-saving operation and maintenance management of this basic infrastructure is an inevitable demand for the supplier of cloud computing (Wibowo et al. 2018).

Presently, users often purchase large amounts of equipment to guarantee peak business operation demands. But for actual operation processes, the load of the equipment is generally low (Mastelic and Brandic 2015), especially in the lowloading period. A long-term low utilization rate will lead to a large waste of resources and energy. 
A cloud-computing data center supports multi-tenant applications of resources. The utilization rate of resources can be effectively improved through the historical statistical information of business, and the coordination of business/resource scheduling management. In typical applications, a cloud-computing data center using energy-saving technology can increase the load of resources to a significantly higher level (Rong et al. 2016), remove the loss in the process of resources' scheduling, and double the resources' payload. During night operations, when the overall load of the data center decreases, the unused resources can be transferred to the idle mode, to maximize the green, low-carbon and energy-saving operation of the data center (Hao et al. 2012).

(4) Privacy and security. In the cloud-computing environment, the centralized and large-scale management of basic resources shifts the security problems to the server side in the data center. From the specialization perspective, end users can achieve business security through the security mechanism of the cloud data center, without consuming too much resources and power (Jin et al. 2014; Sen 2015). At the same time, cloud-computing centers will be directly responsible for the security of all users and specifically focus on the main security risks including data access risk, data storage risk, information management risk, data isolation risk, legal investigation support risk, as well as sustainable development and migration risk.

The security control of cloud computing can be integrated by the basic hardware and software security design. The architecture, strategy, authentication, encryption, and other aspects of a cloud-computing system ensure the information security of cloud-computing servers.

Cloud computing reduces the risk of data loss or leakage from individuals by storing data in a centralized database (Chang and Ramachandran 2015). At the same time, a cloud-computing center also uses a variety of backup methods in security and disaster recovery to guarantee that data will not be lost or illegally tampered with.

\subsubsection{Urban Heat Island Use Case}

Remote-sensing data analysis of a large area is a traditional approach to extract temperature information of cities for UHI modeling and prediction. Google Earth Engine (GGE) is a cloud-based platform sharing large numbers of satellite data online and allowing data analysis and processing on the fly (Gorelick et al. 2017).

Chakraborty and Lee (2019) implemented the SUE algorithm on the Google Earth Engine platform using MODIS images to calculate the UHI intensity for over 9500 urban clusters using over 15 years of data, making this one of the most comprehensive characterizations of the surface UHI to date. They designed an interactive, publicfacing Web application to query UHI intensities of almost all urban clusters based on GGE. Ravanelli et al. (2018a,b) took advantage of GGE and the Climate Engine (CE) tool to process the huge amount of satellite Earth observation data (6000 Landsat 
images) over the period of 1992-2011 and realized wide spatiotemporal monitoring of surface UHI and its connection with land cover changes. Yu et al. (2019) utilized cloud-based computing of spatial and landscape analysis to identify the multi-scale spatiotemporal patterns and characteristics of regional heat islands.

Cloud-computing techniques enable researchers to calculate geophysical parameters from large numbers of remote-sensing data with high and efficient performance. The cloud-computing platform, like Google Earth Engine, assists users to store and manage original raw datasets and provides interactive SaaS for customized algorithms deployment and running for specific UHI-related use cases. These functions are successful in addressing the computing challenges of big data handling, efficiency, computing-intensive modeling and processing, and data security.

\subsection{Edge Computing for Smart Cities}

\subsubsection{Methodology}

With the development of computation technology and hardware, a large number of smart devices are integrated with sensors, enabling them to acquire real-time data and information from the environment. This phenomenon has culminated in the captivating concept of the IoT in which all smart things, such as smart cars (Morabito et al. 2018), wearable devices (Chen et al. 2017), sensors and industrial and utility components (Mehta et al. 2018) are connected via networks and empowered with data analytics that are significantly changing the way we work, live, and play. In the past few years, many scientific and industrial organizations have introduced and implemented the concept of IoT in various fields such as smart homes, smart cities, smart traffic, and smart environments. Edge computing is a new paradigm in which extensive computing and storage resources are placed to provide cloud-computing capabilities at the edge (variously referred to as cloudlets or micro data centers) of the Internet (Satyanarayanan 2010). Edge computing is a mesh network of micro-data centers that process or store data locally and push all received data to a centralized data center or cloud-storage repository (Butler 2017). By implementing computation closer to the edge of the network, analytics of complex data can be realized in nearreal time. In applications, the forms of edge are various; for example, a gateway at a smart home is the edge between home devices and the central cloud; a micro-data center and a cloudlet are the edge between a smartphone and the central cloud.

The main function of edge computing is to ingest, store, filter, and send data to the central cloud systems ("What Is Edge Computing?|GE Digital" n.d.). At the heart of a smart city, there is widespread deployment of IoT sensor networks, which provide a regular flow of data that allows for effective and efficient management of services and assets. Typical deployment scenarios include a large scope of content: from bus tracking to traffic light management, street lighting control, air quality, and pollution monitoring. We envision that edge-computing could have similar impact on 
our society as that of cloud computing. Edge computing provides new possibilities in IoT applications, particularly for those tasks relying on AI techniques such as object detection (Ananthanarayanan et al. 2017), face recognition (Hu et al. 2016), language processing (Lewis et al. 2014), and obstacle avoidance (Zhang and Ye 2016).

\subsubsection{Challenges, Motivations, and Opportunities}

Nowadays, a smart city relies on the infrastructure of edge computing to leverage most of the up-to-date data-driven technologies. With edge computing, services can be ensured to flow continuously through local data processing even when the Web connection is interrupted (Abbas et al. 2017). For example, driverless cars and other modern IoT devices are designed to be built with enough processing capability, so that they can perform some of the computation themselves at the edge, without sending it to the central cloud. Edge-computing technology provides an attractive and resilient platform for cities, while at the same time reducing backhaul costs (Tran et al. 2017a, b), both in terms of the amount of data required and the sharing of connections by creating a mesh network.

There are challenges both in the big data generated and in creating the necessary network infrastructure to support an increasing number of end devices. Edge computing offers a solution to many of the challenges described in Sect. 41.2.2, which opens up many possibilities for smart cities. According to the advantages discussed above, edge computing can contribute to the following computing challenges of smart cities:

(1) Latency and efficiency. In a high-efficiency computing system, any device connected to the Internet has to be responsive in a short period of milliseconds. Any lag in the communication between network and devices is termed latency. Edge computing can eliminate the latency issue as it works on the principle of a more distributed network. This kind of system has the capability to guarantee real-time information processing and maintains a more reliable network (Hu et al. 2015). On the other hand, edge-computing processes the massive data generated by different types of IoT devices at the edge of network, instead of transmitting them to the centralized cloud infrastructure. Therefore, edge computing can provide services with faster response and greater quality in comparison with cloud computing, which greatly improves the efficiency of collecting, transferring, processing, and analyzing data generated by arrays of IoT devices.

(2) Privacy and security. Security concerns are more related to the transfer of data over a network to the central cloud. In an edge architecture, any outage would be limited to the edge devices and local applications. Therefore, edge computing will improve privacy and security by omitting the transmission since the data are stored and processed in or closer to the edge devices (He et al. 2018). With the improvement of authentication technology, the privacy and security 
of edge computing can be further guaranteed by the emergence of biometric authentication such as fingerprint authentication, face authentication, touchbased, or keystroke-based authentication (Yi et al. 2015; Zhou et al. 2017).

(3) Internet load reduction. According to the Cisco Global Cloud Index ("Cisco Global Cloud" n.d.), the amount of traffic running through cloud-computing networks will increase to 14.1 zettabytes per year in 2020. This immersive amount of traffic can be removed from the central cloud by processing some of the data closer to the edge. Additionally, moving the processing of data away from the central cloud can minimize the network burden where the Internet bandwidth is limited (Lyu et al. 2018).

(4) Sustainability. Edge-computing systems provide the capability of decentralizing computation power, which support fault tolerance in that when one of the edge devices fails, other nodes and associated IT assets will still remain operational (Ning et al. 2019). This concept is similar to the cloud disaster recovery strategy ("Disaster Recovery Planning GuidelArchitectures," n.d.) by using multiple available zones and regions to ensure that the data and applications are not lost in a catastrophic event.

Edge computing introduces a new concept that computing should happen as close as possible to the data sources. With this architecture, a request could be generated from the top of the computing paradigm and processed at the edge. By deploying edge computing, software engineers can create additional applications that utilize edgecomputing platforms to leverage existing technology and benefit the smart cities in the following ways ("Smarter Cities with Edge Computing" n.d.):

(1) Streetlighting. A number of cities are in the process of upgrading their streetlights to lower-power LEDs. With the major cost of these upgrades being the physical fitting, edge appliances can be added to provide lighting controls (Xing et al. 2018).

(2) Security cameras. Nowadays, CCTV cameras have been a critical tool in modern policing systems. Edge computing can allow low-cost wireless IP cameras to be deployed in these systems, which will offer considerably less cost (Yi et al. 2017).

(3) Health emergency and public safety management. For applications that require real-time prediction and low latency such as health emergencies (Wang et al. 2017) and public safety (Zhang and Ye 2016) management, edge computing is also an appropriate paradigm since it could save the data transmission time as well as simplify the network structure. Decisions and diagnosis could be made and distributed from the edge of the network, which is more efficient compared with collecting information and making decisions at a central cloud.

(4) Location awareness. For geoinformatics-based applications such as transportation and utility management, edge computing exceeds cloud computing due to location awareness (Shi et al. 2016). In edge computing, data could be collected and processed based on geographic location without being transferred to the central cloud. 


\subsubsection{Urban Heat Island Use Case}

Unlike cloud computing, edge devices are commonly decentralized. In order to monitor UHI from distributed sensors, edge computing offers closer contacts to each individual sensor, thus reducing energy consumption and response time during the transfer of observation data (Ngoko et al. 2018). Edge devices are those mounted directly on the edge for urban sensing of properties such as microclimate, having better durability compared to wireless devices. Densely distributed buildings in urban areas work as an ideal candidate for the deployment of edge devices, providing close proximity to the UHI impact factors such as temperature, humidity, and wind speed. Due to climate change, heating and cooling consume significant energy in buildings. These sectors contribute greatly to UHI and can be monitored by smart building sensors (Seitz et al. 2017). Lightweight tasks like data cleaning and basic decision support can be performed, and therefore contributes to UHI mitigation. Applications that support edge computing can benefit the field of UHI in: (1) allowing users to browse and query the UHI of cities around the world from a gateway; (2) providing a means to access real-time datasets from the edge without any latency; and (3) allowing users to search for a city of interest, query cities to generate charts of seasonal and long-term surface UHI, and download the UHI data.

\subsection{Mobile Computing for Smart Cities}

\subsubsection{Methodology}

Mobile computing could be described as a form of human-computer interaction where the computer is portable and transported during normal usage (Qi and Gani 2012; Akherfi et al. 2018). The fundamental concepts of mobile computing include: (1) communication, (2) hardware, and (3) software. Specifically, the communication concept refers to the wireless networks, data traffic, and protocols. The hardware could be any type of mobile device, which includes: (1) laptops, (2) tablets, (3) smartphones, (4) carputer, and others. The category boundaries of such devices are blurry, as more and more portable devices are installed with microchips and wireless modules, all of which have some computing power and the ability to transfer data through networks as a part of the mobile-computing hardware (Tong et al. 2016). The software in mobile computing consists of the applications in mobile device hardware, such as customized industry software, data collection applications, and Web browsers.

In the past decade, mobile computing has developed in two ways (Kumar et al. 2013): (1) deployment of sensors, and (2) growth in smartphones. It was also challenged by the explosion of big data (Laurila et al. 2012). Different from purposeoriented IoT, mobile devices are integrated with multi-purpose sensors, such as GPS receivers, accelerometers, gyroscopes, and microphones. With the growth in both 
smartphone technologies and number of users, mobile devices are transitioning from specialized and customized platforms to powerful computing interfaces (Al-Turjman 2018). Mobile computing itself is also becoming a computing offloading contributor. The application layer of mobile computing faces various challenges due to its features. However, with the fast growth in communication technologies, including 4G and 5G networks and high-speed city Wi-Fi (Tran et al. 2017a, b), and mobile technologies in general, the number of applications running on mobile devices is growing at an exponential rate.

\subsubsection{Challenges, Motivations, and Opportunities}

In addition to most computing architectures in a wired network, mobile computing is different in the following aspects (Qi and Gani 2012): (1) Mobility: mobilecomputing nodes or devices are expected to be portable and transportable; the computing power is not physically limited to a certain location and follows the principle of bringing computing to the data instead of transferring the data to computing resources. (2) The diversity of network conditions: the networks that mobile devices use are often not fixed; communication could be achieved through high-bandwidth or low-bandwidth networks; and the mobile device may even operate offline. (3) Inconsistency: as mobile devices are limited by their battery power and wireless network conditions, the inconsistency of communication and change of working status are expected and requires the mobile devices to switch modes to adapt to specific situations. (4) Asymmetric communication: wireless networks are often set with different bandwidths for downlink and uplink, which causes asymmetric communications between backend servers and local devices. (5) Low reliability: wireless communications are susceptible to interference; the security issues are enlarged in such networks and affect the reliability of mobile computing (Qi and Gani 2012).

The rapid development of mobile computing and smartphone applications is enabling integrated growth of smart-city applications. As stated in Sect. 41.2.2, mobile computing can help to improve the following challenges of smart-cities computing:

(1) Satisfy the need of users from different areas. Mobile computing supports smartcity computing in the forms of mobility and flexibility, which could help both end users and policy makers to meet different computing demands in different scenarios. Application use cases include services in higher education (Gikas and Grant 2013), and location-based services in general, which all utilize the mobility side of smart devices and allow them to act as both a data collector and data user (Raja et al. 2018). Another application of mobile computing is to utilize and integrate smart devices in smart spaces (Zheng and Ni 2010). The concept of the smart city is a big domain with enough space for the expansion and adaptability of mobile computing. Research topics including dynamic offloading for 
mobile devices (Huang et al. 2012) and mobile cloud computing are all interactive examples of smart devices in smart spaces. Mobile cloud computing has been envisioned since 2009 as a combination of cloud computing and mobile computing, which leverages the mobility side of mobile computing and integrates with the elastic computing power from cloud computing (Tong et al. 2016; Dinh et al. 2013; Fernando et al. 2013). When integrated with cloud-computing power, it could also serve as an edge-computing device in the cloud-computing network.

(2) Computing efficiency and near-real-time analysis and feedback. Smart device holders are often fed with various information or data through sensors on the smart devices; with mobile-based computing power, stream-like data flow could be analyzed locally and uploaded to the centralized databases at the same time. End users with smart devices on hand could get feedback or results immediately; routing and mapping services, language translation services, and instant weather services are all good examples of this (Talukdar 2010). At the same time, publicsecurity services and danger-awareness services could also be provided through mobile computing and locally based services (Aubry et al. 2014), such as the lost child and healthcare applications discussed in Sect. 41.1.2. The challenges in smart-city implementations bring new motivations and opportunities for the development of mobile computing and vice versa.

As one of its important components, mobile computing is enhancing the smartcity experience in the following aspects: (1) Transport and traffic management for both personal end users and policy makers; (2) Utilities and energy monitoring across the network, and (3) Improving public safety and smart-city security awareness.

\subsubsection{Urban Heat Island Use Case}

Mobile computing and mobile-based technologies are integrating innovative concepts and ideas to increase UHI awareness and aid city design to reduce the UHI effect. As Wong et al. (2014) mentioned in their reviews, tools have been developed and implemented to allow users to gather instantaneous energy performance feedbacks on their decisions and plans of building designing, such as the building orientation and thermal performance, through mobile-based applications (i.e., iPad/smartphone application). At the same time, mobile devices provide volunteered geographic information (VGI) to enhance the near-real-time estimation of UHI. For example, Koukoutsidis (2018) utilized mobile crowdsensing to estimate the mean area temperature in a linear region that exhibits the UHI effect. 


\subsection{Case Study}

\subsubsection{Urban Heat Island (UHI)}

The direct cause of UHI is urbanization, which leads to the loss of more vegetation and causes more surfaces to be paved or covered with impervious materials such as cement, asphalt, buildings, and walls. Challenges are revealed due to the complexity of the composition of UHI impact factors. Major ones are stated by Oke (1982) in his previous studies and include: (1) the inherent complexity of the city-atmosphere system; (2) the lack of clear conceptual and theoretical frameworks; and (3) the expense and difficulty of observation in cities. UHI is a very common challenge to all urban areas in the world, although in megacities it is serious and less so in small towns.

UHI is usually measured in three scales: boundary UHI, canopy UHI, and surface UHI. Boundary UHI is measured from the altitude of the rooftop to the atmosphere. It is generally used to investigate the UHI effect at mesoscale and is acquired by using, for example, radiosondes. Canopy UHI is measured at the altitude that ranges from the ground surface to the rooftop. An assessment of canopy UHI is most suitable for a microscale study and is generally derived based on weather station data. Surface UHI is measured at the Earth surface level. Researchers have often used satellite images (e.g., thermal bands of Landsat TM/ETM/OLI, MODIS, AVHRR) to obtain the effect of surface UHI (Zhang et al. 2009). Researchers used remotely sensed data and stationary meteorological monitoring data to analyze the UHI changes and effects in the long or short term (Earl et al. 2016), as well as the relationship between UHI and land cover changes (Chen et al. 2006; Charkraborty and Lee 2019). A lot of research has simulated and evaluated UHI and its effect on the future by using numerical modeling based on real-time meteorological data (Morris et al. 2015).

\subsubsection{UHI Challenges and Opportunities}

From the aforementioned scientific challenges, UHI introduces its own computing challenges, mostly concentrated on handling the aspects of the expense and difficulty of observation in cities. These challenges include: (1) management of heterogeneous data sources; (2) integration of a huge volume of remotely sensed data and real-time meteorological data; and (3) a large amount of computation in modeling, visualizing, simulating, and predicting. Cloud computing has existed in the long term for allocating computing resources to enable the auto-scalable modeling and detecting in many study fields and has proved to be an efficient and economical solution (Yang et al. 2017a). Google Earth Engine is a cloud-computing platform, offering intrinsically parallel computational resources, and enabling monitoring and measurement of changes in the Earth's environment, at planetary scale, on a large catalog of Earth observation data (Moore and Hansen 2011). An implementation of 
large-scale correlation between land surface temperature and land cover alteration research is conducted upon this platform and has illustrated the capability of using cloud computing for efficient UHI monitoring (Ravanelli et al. 2018a, b).

The emergence of $5 \mathrm{G}$ and IoT technologies in the current era is bringing opportunities to facilitate advances in urban microclimate study with finer spatiotemporal resolution beyond just satellite imagery analysis (Li et al. 2018). Voogt and Oke (2003) argued that thermal remote sensors have a credible ability to observe the surface UHI and require consideration of the intervening atmosphere and surface radiative properties, leading to extra conversions and corrections. With implementing sensor device networks directly into the environment, urban environmental factors like air temperature are more accurately measured. These sensor networks can be designed and implemented for advanced urban microclimate and environment modeling (Jha et al. 2015). Challenges follow when considering the real-time streaming nature of IoT, as it requires the capacity of ingesting the large number of data and producing results with higher speed that is beyond the capability of conventional architectures (Rathore et al. 2018). Santamouris (2015) analyzed heat island magnitude and characteristics in one hundred cities and regions and indicated that analysis of $43 \%$ station measurements are only based on one station from urban and one from rural. According to the Gartner, up to 20.4 billion IoT devices will be connected machineto-machine by 2020 (Meulen 2017), offering great potential to increase the number of sensors utilized for UHI research.

Since the first time it was introduced by Howard (1818), in the past 200 years, numerous studies have been developed to model UHI intensity, simulate, and predict UHI effects. However, it was proved from analyzing one hundred Asian and Australian cities and regions, that a systematic analysis like a workflow is still needed (Santamouris 2015). Coupling with aforementioned computing techniques (cloud computing, edge computing, and mobile computing), the following introduces a theoretical integrated workflow to enable the efficient data storage and processing for handling urban informatics challenges and using UHI as an example. This workflow targets the last two scientific challenges of UHI, and the overall architecture is illustrated in Fig. 41.4, starting from collecting urban observation data with mobile devices to the centralized cloud-based data analysis, and finishing with generating intelligent supportive materials for UHI monitoring and managing.

\subsubsection{Integrated Workflow}

\subsubsection{Mobile Computing for Local Fast Response}

Data in Fig. 41.4 are directly collected by sensors within a large sensor network deployed in the urban environment. Data streams into the workflow by entering the first gate: mobile computing. In general, the capacity of mobile devices is low, and due to the limitations like battery life, only lightweight preprocessing like data cleaning and reorganizing can be performed at the mobile computing stage. However, in situ 


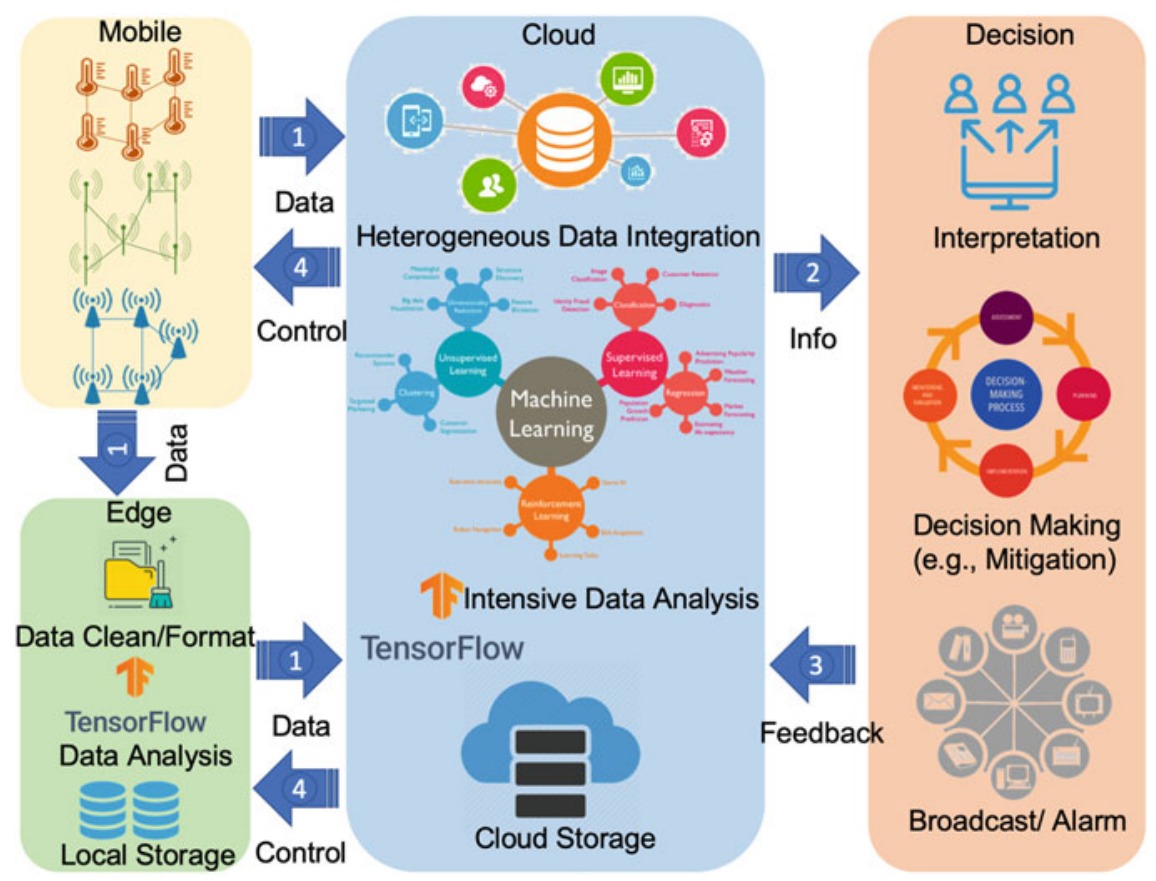

Fig. 41.4 Overall architecture of computing for UHI

monitoring coupled with light data understanding can reduce time latency for jobs that do not require extensive computation but only the ability to make simple judgments. For instance, alarms setup on a mobile device with constrained temperature threshold can be triggered responsively when unexpected heat is detected. Though the computing capabilities of mobile devices are low, with hundreds and thousands of contributions from them, appreciable computational resources are preserved for more intensive works like microscale UHI modeling (Mirzaei 2015).

\subsubsection{Edge Computing for Data Preprocessing and Direct Microcontrol}

Besides collecting data on the edge and passing the raw data to the cloud like mobile computing, edge computing offers more capacities for better data preprocessing. With the increasing data volume, uploading everything raw to the cloud can take a significant amount of time, and the heavy duty that is loaded to the center cluster can exceed the limit of the computing resources. To fill the gap between mobile computing and cloud computing, enhancing the performances regarding response time, data transform, data safety, and privacy, edge computing is integrated to the workflow to allow downstream data representing cloud services and upstream data 
representing IoT services (Sun and Ansari 2016; Shi et al 2016; Yannuzzi et al. 2014). Similarly, works that do not require much computation can be done directly from the edge and provide feedbacks to the sensors to reduce time lag (Gerla 2012). Data from the Array of Things (AoT) (University of Chicago 2019; see Sect. 4.7) project at the University of Chicago monitors local temperatures and other environmental elements from networks composed of hundreds of sensors, providing observations with the resolution of seconds. The high-velocity data transfers within the network can cause traffic congestion due to the limited bandwidth. The Google cloud platform supports edge computing with AI, enabling potential real-time data analytics (Google 2019).

\subsubsection{Cloud Computing for Massive Data Processing and Analytics}

Like every big data problem, a sensor dataset at fine temporal resolution for UHI monitoring (e.g., streaming AoT data) introduces a data storage challenge. Cloud computing as the final layer of UHI data processing and analyzing has been well studied for enabling heavy computations by transferring big data storing and processing from a local to a centralized cluster (Yang et al. 2017b). Empowered with the auto-expandable nature of the virtual storage mechanism, data streamed from sensors transfer through edges to the center for better management. With the wellresourced computing capacity, the cloud cannot only process the data that mobile and edge devices cannot, but also accelerate the processing beyond a standalone server.

IoT networks are massive and can be distributed with different protocols established by different management departments. Therefore, UHI-related attributes like temperature, humidity and wind speed from different networks are potentially captured with sensors powered by different standards. Data heterogeneity is one of the major concerns and the massive data cleaning workload requires significant computational capability. The cloud as a centralized computing resource pool offers sufficient capacity for such workload (Botta et al. 2014). As mentioned, there are many factors contributing to UHI study. Changing the composition leads to requirements for model parameter adjustments. SaaS as introduced in Sect. 41.3.1 and provided with cloud computing allows users to duplicate a model directly from a current version and customize the new one to fit the new environment. Advantages include reduced model-building time and decreased human error when transferring the experimental environment.

\subsubsection{Mobile-Edge-Cloud Integrated Computing for UHI}

A weather forecast example provided by a previous study indicated the basic workflow when the simulation is decomposed into a process-oriented pipeline (Tsahalis et al. 2013). Weather research shares conceptual similarities to UHI, and thus, their example is applied here as a base version of the conventional workflow. Heusinkveld et al. (2010) carried out an assessment of UHI intensity in Rotterdam using an innovative mobile bio-meteorological measuring platform mounted on a cargo bicycle. 
Physiologically equivalent temperatures were calculated directly from the measurements and the intensity of UHI was evaluated in real time. Coupling with the IoT and mobile devices empowered a real-time urban microclimate analysis framework that integrated with the sensor network and cloud computing (Rathore et al. 2018); our workflow gains the experience from both. This enhanced framework composed of cloud computing, edge computing, and mobile computing is able to successfully address the previously introduced UHI challenges. Starting from measuring the geographic environmental of ground, air, and water, mobile computing can directly sense these parameters and give a quick response (e.g., a UHI detection alarming system) with minor data manipulation before entering the major processing and modeling procedures. Edge computing offers a higher computational capacity, mitigating the heavy workload that is initially carried by the centralized module. Buildingscale UHI (i.e., building energy model) is limited to the study of an isolated building, requiring less computational resources as it considers less neighborhood environmental impacts (Mirzaei 2015). Therefore, UHI modeling, visualizing, simulating, and predicting for a smaller UHI study scale (i.e., building scale) can be directly computed on the edge for more efficiency. There are many tasks that cannot be satisfied with the limited resources from mobile computing or edge computing, such as heterogeneous data integration, and larger scale (e.g., microclimate) UHI modeling. The cloud as a big centralized resources pool is powered with enormous computing capabilities. UHI-related observation data like temperature, humidity, and windspeed are transferred from sensors to the cloud after a certain effort made by mobile computing and edge computing for data cleaning and preprocessing. Heterogeneous data integration on the cloud will be triggered for the massive data coupled with mixed data types and data standards. Large-scale UHI modeling, simulating, etc., are performed within the cloud. Elasticity that is offered as one of the key features of the cloud dispatches computing resources on demand and surpasses the traditional method of using a single computer for analysis, saving resources while providing enough capacity for the heavy tasks. All three computing paradigms work seamlessly from getting the sensor data to processing, analyzing, and decision support, enabling an efficient and effective workflow as a whole to handle the UHI challenges.

These three computing components should be leveraged and kept in balance when applied to UHI monitoring, data analysis, and problem solving. For instance, deploying edge nodes with higher computing capacity may increase the operational cost for processing the IoT data streams compared to processing them in the centralized cloud (Sun and Ansari 2016). Understanding the tradeoffs among the different interfacings of the three is crucial for maximizing the workflow efficiency and optimizing the computing architecture design. Many other smart-city applications are encountering similar problems, and the demonstrated UHI analytical workflow can be broadly applied when integrating computing components. 


\subsection{Summary}

This chapter introduced the contribution and recent advances of computing for smart cities. The general challenges of computing in smart cities were introduced and include heterogeneous sources of big data, resulting from the unprecedented number of smart sensors and devices, various needs from users in multiple domains, data security, sustainability, and efficiency. To address the challenges, cloud computing, edge computing, and mobile computing were discussed for their advantages and limitations in smart-city applications. Cloud computing provides a unified and efficient platform, large-scale base infrastructure, sustainable and green software and hardware development and addresses system security and recovery issues. Edge computing helps reduce observation latency and increase the efficiency of data collection, improve data privacy and security, reduce data transmission load on computer network, and provide a sustainable decentralization of computing needs. Mobile computing contributes to the smart city with computational mobility and flexibility, and computing efficiency and near-real-time analysis. The characteristics of different computing paradigms were exemplified in the case study of urban heat island. With multiple computing paradigms leveraged, smart-city applications and services can be provided in a more efficient and effective fashion.

\subsubsection{The Future of Urban Computing for Smart Cities}

Big data and IoT are labeled as the primary drivers for the cloud, edge, and mobile computing. The development of mobile computing is increasing at an accelerating speed. With the fast implementation of $5 \mathrm{G}$ networks and closer integration with cloud computing, the mobile-computing system is merging with the cloud-computing network and serving as the network edge. The phrase mobile cloud computing has been frequently referenced in the mobile-computing field (Fernando et al. 2013; Akherfi et al. 2018). When the mobility of mobile computing interacts with the elastic computing power from cloud computing, it will push the whole computing network to a new decentralized computing stage and accelerate the smart-city process. Smarter devices, faster networks, and longer battery lives are the foreseeable future; the transformation of mobile computing and interaction with other computing fields will be the norm.

With the increasing number of mobile devices (phones, drones, cars, etc.), the need for interaction with nearby edge resources will become apparent. Coupled with better processing, computing, and power capacity, as well as the decentralized characteristic of mobile computing, edge computing is expected to provide significantly improved throughput, better performance, and real-time responses, moving both computing and data closer to the user and customizing the processing requirements from each user. Edge computing and mobile computing are both capable of handling localized 
data for fast action for a certain range of area size. However, the increasing urban data volume and cross-city geo-analysis are also driving centralized cloud computing.

Ever since the infrastructure was developed for cloud computing, the combined use of private and public clouds is engaged for many more individual and business purposes. As a mature platform to integrate powerful computing capabilities, large data storage and on-demand data analysis, cloud computing will lead cities toward a smart age - an age based on fully connected, interactive decisionsupporting environment. Within the smart city, a variety of devices (e.g., domestic appliances and semiautomatic vehicles) will connect to the cloud-based Internet for sensing, recording, sharing, and analyzing numerous human-related activities. Coupled with the help from artificial intelligence algorithms, cloud computing will serve companies, governments, and individual residents with smarter solutions.

\section{References}

Abbas N, Zhang Y, Taherkordi A, Skeie T (2017) Mobile edge computing: a survey. IEEE Internet of Things J 5(1):450-465

Akherfi K, Gerndt M, Harroud H (2018) Mobile cloud computing for computation offloading: issues and challenges. Appl Comput Inform 14(1):1-16

Altman SH, Sivo NT, Jacobstein M, Knapp BR, Potter JF, Pernikoff TB (2015) Secure and private location sharing for location-aware mobile communication devices. U.S. Patent 8,989,778

Al-Turjman F (2018) Mobile couriers' selection for the smart-grid in smart-cities' pervasive sensing. Future Gener Comput Syst 82:327-341

Amirebrahimi S, Rajabifard A, Mendis P, Ngo T (2016) A framework for a microscale flood damage assessment and visualization for a building using BIM-GIS integration. Int J Digital Earth 9(4):363-386

Ananthanarayanan G, Bahl P, Bodík P, Chintalapudi K, Philipose M, Ravindranath L, Sinha S (2017) Real-time video analytics: the killer app for edge computing. Computer 50(10):58-67

Anon nd (2019) Array of things file browser. [Online]. https://aot-file-browser.plenar.io. Accessed on 10 May 2019

Anthopoulos LG (2015) Understanding the smart city domain: a literature review. Trans-forming city governments for successful smart cities. Springer, Cham, pp 9-21

Aubry E, Silverston T, Lahmadi A, Festor O (2014) CrowdOut: a mobile crowdsourcing service for road safety in digital cities. In: 2014 IEEE international conference on pervasive computing and communication workshops (PERCOM WORKSHOPS), pp 86-91

Bera S, Misra S, Rodrigues JJ (2015) Cloud computing applications for smart grid: a survey. IEEE Trans Parallel Distrib Syst 26(5):1477-1494

Bibri SE (2018) The IoT for smart sustainable cities of the future: an analytical framework for sensor-based Big Data applications for environmental sustainability. Sustain Cities Soc 38:230 253

Botta A, De Donato W, Persico V, Pescapé A (2014) On the integration of cloud computing and internet of things. In: 2014 international conference on future internet of things and cloud, pp 23-30

Butler B (2017) What is edge computing and how it's changing the network. Netw World 21(09)

Casanueva A, Burgstall A, Kotlarski S, Messeri A, Morabito M, Flouris AD, Nybo L, Spirig C, Schwierz C (2019) Overview of existing heat-health warning systems in Europe. Int J Environ Res Public Health 16(15):2657 
Cascetta E, Carteni A, Pagliara F, Montanino M (2015) A new look at planning and designing transportation systems: A decision-making model based on cognitive rationality, stake-holder engagement and quantitative methods. Transp Policy 38:27-39

Chakraborty T, Lee X (2019) A simplified urban-extent algorithm to characterize surface urban heat islands on a global scale and examine vegetation control on their spatiotemporal variability. Int J Appl Earth Obs Geoinf 74:269-280

Chang V, Ramachandran M (2015) Towards achieving data security with the cloud computing adoption framework. IEEE Trans Serv Comput 9(1):138-151

Chen XL, Zhao HM, Li PX, Yin ZY (2006) Remote sensing image-based analysis of the relationship between urban heat island and land use/cover changes. Remote Sens Environ 104(2):133-146

Chen Z, Hu W, Wang J, Zhao S, Amos B, Wu G, Ha K, Elgazzar K, Pillai P, Klatzky R, Siewiorek D (2017) An empirical study of latency in an emerging class of edge computing applications for wearable cognitive assistance. In: Proceedings of the second ACM/IEEE symposium on edge computing, p 14

Cisco Global Cloud Index Projects Cloud Traffic to Nearly Quadruple Representing 92 Per-cent of Total Data Center Traffic by 2020 [WWW Document], n.d. URL https://newsroom.cisco.com/ press-release-content?type $=$ webcontent $\&$ articleId $=1804748$. Accessed on 13 May 2019

Conti S, Meli P, Minelli G, Solimini R, Toccaceli V, Vichi M, Beltrano C, Perini L (2005) Epidemiologic study of mortality during the Summer 2003 heat wave in Italy. Environ Res 98(3):390-399

Dai YS, Yang B, Dongarra J, Zhang G (2009) Cloud service reliability: Modeling and analysis. In: 15th IEEE pacific rim international symposium on dependable computing, pp 1-17

David R (2018) Smarter cities with edge computing. https://www.smartcitiesworld.net/opinions/ opinions/smarter-cities-with-edge-computing

Davies M, Steadman P, Oreszczyn T (2008) Strategies for the modification of the urban climate and the consequent impact on building energy use. Energy Policy 36(12):4548-4551

Dinh HT, Lee C, Niyato D, Wang P (2013) A survey of mobile cloud computing: architecture, applications, and approaches. Wirel Commun Mobile Comput 13(18):1587-1611

Disaster Recovery Planning GuidelArchitectures [WWW Document], n.d. Google Cloud 5.13.2019. https://cloud.google.com/solutions/dr-scenarios-planning-guide

Earl N, Simmonds I, Tapper N (2016) Weekly cycles in peak time temperatures and urban heat island intensity. Environ Res Lett 11(7):074003

EPA (2016) Climate Change Indicators in the United States, 2016. Available online: https://www. epa.gov/climate-indicators. Accessed on 8 April 2019

Etro F (2015) The economics of cloud computing. In: Cloud technology: concepts, methodologies, tools, and applications, pp 2135-2148. IGI Global

Fernando N, Loke SW, Rahayu W (2013) Mobile cloud computing: a survey. Future Gener Comput Syst 29(1):84-106

Filleul L, Cassadou S, Médina S, Fabres P, Lefranc A, Eilstein D, Le Tertre A, Pascal L, Chardon B, Blanchard M, Declercq C (2006) The relation between temperature, ozone, and mortality in nine French cities during the heat wave of 2003. Environ Health Perspect 114(9):1344-1347

Fu Z, Sun X, Liu Q, Zhou L, Shu J (2015) Achieving efficient cloud search services: multi-keyword ranked search over encrypted cloud data supporting parallel computing. IEICE Transac Commun 98(1): 190-200

Gerla M (2012, June) Vehicular cloud computing. In: 2012 The 11th annual mediterranean ad hoc networking workshop (Med-Hoc-Net), pp 152-155. IEEE

Giannaros TM, Melas D, Daglis IA (2014) Development of an operational modeling system for urban heat islands: an application to Athens, Greece. Natural Hazards Earth Syst Sci 14(2):347358

Gikas J, Grant MM (2013) Mobile computing devices in higher education: Student perspectives on learning with cellphones, smartphones and social media. Internet Higher Educ 19:18-26

Gong C, Liu J, Zhang Q, Chen H, Gong Z (2010) The characteristics of cloud computing. In: 39th international conference on parallel processing workshops, pp 275-279 
González-Gil A, Palacin R, Batty P, Powell JP (2014) A systems approach to reduce urban rail energy consumption. Energy Convers Manage 80:509-524

Google (2019) AI at the edge. [Online] Accessed on 10 May 2019. https://cloud.google.com/edgetpu/

Gorelick N, Hancher M, Dixon M (2017) Google Earth Engine: planetary-scale geospatial analysis for everyone. Remote Sens Environ 202:18-27

Graham S, Marvin S (2002) Telecommunications and the city: electronic spaces, urban places. Routledge.

Guest CS, Willson K, Woodward AJ, Hennessy K, Kalkstein LS, Skinner C, McMichael AJ (1999) Climate and mortality in Australia: retrospective study, 1979-1990, and predicted impacts in five major cities in 2030. Climate Res 13(1):1-15

Haines A, Kovats RS, Campbell-Lendrum D, Corvalán C (2006) Climate change and human health: impacts, vulnerability, and mitigation. Lancet 367(9528):2101-2109

Hameed K (2003) The application of mobile computing and technology to health care services. Telematics Inform 20(2):99-106

Hao L, Lei X, Yan Z, ChunLi Y (2012) The application and implementation research of smart city in China. In: 2012 international conference on system science and engineering (ICSSE), pp 288-292

Hashem IAT, Yaqoob I, Anuar NB, Mokhtar S, Gani A, Khan SU (2015) The rise of "Big Data" on cloud computing: review and open research issues. Inf Syst 47:98-115

Hashim Raza Bukhari S, Siraj S, Husain Rehmani M (2018) Wireless sensor networks in smart cities: applications of channel bonding to meet data communication requirements. In: Transportation and power grid in smart cities: communication networks and services, pp 247-268

He D, Chan S, Guizani M (2018) Security in the internet of things supported by mobile edge computing. IEEE Commun Mag 56(8):56-61

Heusinkveld BG, Van Hove LWA, Jacobs CMJ, Steeneveld GJ, Elbers JA, Moors EJ, Holtslag AAM (2010) Use of a mobile platform for assessing urban heat stress in Rotterdam. In: Proceedings of the 7th conference on biometeorology, vol 20, pp 433-438

Hoffiman J (2018) Where do we need shade? Mapping urban heat islands in Richmond, Virginia. https://www.climate.gov/news-features/climate-case-studies/where-do-we-need-shade-map ping-urban-heat-islands-richmond

Hondula DM, Georgescu M, Balling RC Jr (2014) Challenges associated with projecting urbanization-induced heat-related mortality. Sci Total Environ 490:538-544

Howard L (1818) The climate of London, vol 1. W. Phillips, sold also by J. and A. Arch

Hu YC, Patel M, Sabella D, Sprecher N, Young V (2015) Mobile edge computing—a key technology towards 5G. ETSI White Paper 11(11):1-16

Hu W, Gao Y, Ha K, Wang J, Amos B, Chen Z, Pillai P, Satyanarayanan M (2016) Quantifying the impact of edge computing on mobile applications. In: Proceedings of the 7th ACM SIGOPS Asia-Pacific workshop on systems, p 5

Hu F, Yang C, Jiang Y, Li Y, Song W, Duffy DQ, Schnase JL, Lee T (2018) A hierarchical indexing strategy for optimizing Apache Spark with HDFS to efficiently query big geospatial raster data. Int J Digital Earth 1-19

Huang D, Wang P, Niyato D (2012) A dynamic offloading algorithm for mobile computing. IEEE Trans Wirel Commun 11(6):1991-1995

Hwang JS, Choe YH (2013) Smart Cities Seoul: a case study. ITU-T technology watch report. pp $1-20$

Jadeja Y, Modi K (2012) Cloud computing concepts, architecture and challenges. In: 2012 international conference on computing, electronics and electrical technologies (ICCEET), pp $877-880$

Jha M, Marpu PR, Chau CK, Armstrong P (2015) Design of sensor network for urban micro-climate monitoring. In: IEEE first international smart cities conference (ISC2), pp 1-4

Jin J, Gubbi J, Marusic S, Palaniswami M (2014) An information framework for creating a smart city through internet of things. IEEE Internet Things J 1(2):112-121 
Jonas E, Pu Q, Venkataraman S, Stoica I, Recht B (2017) Occupy the cloud: Distributed computing for the 99\%. In: Proceedings of the 2017 symposium on cloud computing, pp 445-451

Kakderi C, Komninos N, Tsarchopoulos P (2016) Smart cities and cloud computing: lessons from the STORM CLOUDS experiment. J Smart Cities 2(1):4-13. https://doi.org/10.18063/JSC.2016. 01.002

Karwot J, Kaźmierczak J, Wyczółkowski R (2016) Smart water in smart city: A case study. In: Proceedings of SGEM 16th international scientific conference on earth and geosciences 3:851858

Kaushal DS, Khan Y (2014) Cloud computing services in medical healthcare solutions. Int J Res 1(4):312-324

Kofinas DT, Spyropoulou A, Laspidou CS (2018) A methodology for synthetic household water consumption data generation. Environ Modell Softw 100:48-66

Koukoutsidis I (2018) Estimating spatial averages of environmental parameters based on mobile crowdsensing. ACM Transac Sens Netw (TOSN) 14(1):2

Kumar K, Liu J, Lu YH, Bhargava B (2013) A survey of computation offloading for mobile systems. Mobile Netw Appl 18(1):129-140

Lacinák M, Ristvej J (2017) Smart city, safety and security. Procedia Eng 192:522-527

Laurila JK, Gatica-Perez D, Aad I, Bornet O, Do TMT, Dousse O, Eberle J, Miettinen M (2012) The mobile data challenge: Big Data for mobile computing research. In: Proceedings of the international conference on mobile computing

Lehrig S, Eikerling H, Becker S (2015) Scalability, elasticity, and efficiency in cloud computing: a systematic literature review of definitions and metrics. In: Proceedings of the 11th international ACM SIGSOFT conference on quality of software architectures, pp 83-92

Lewis G, Echeverría S, Simanta S, Bradshaw B, Root J (2014) Tactical cloudlets: Moving cloud computing to the edge. In: 2014 IEEE military communications conference, pp 1440-1446

Li Y, Zhou Y, Shi Y, Zhu X (2012) Investigation of a coupling model of coordination be-tween urbanization and the environment. J Environ Manage 98:127-133

Li Y, Yang R, Yang C, Yu M, Hu F, Jiang Y (2017) Leveraging LSTM for rapid intensifications prediction of tropical cyclones. In: ISPRS annals of photogrammetry, remote sensing and spatial information sciences, vol 4

Li S, Da Xu L, Zhao S (2018) 5G Internet of Things: a survey. J Indus Inf Integr 10:1-9

Liebig T, Piatkowski N, Bockermann C, Morik K (2017) Dynamic route planning with real-time traffic predictions. Inf Syst 64:258-265

Liu Y, Shi J, Jian M (2017) Understanding visitors' responses to intelligent transportation system in a tourist city with a mixed ranked logit model. J Adv Transport

Lv Z, Li X, Wang W, Zhang B, Hu J, Feng S (2018) Government affairs service platform for smart city. Future Gener Comput Syst 81:443-451

Lyu X, Tian H, Jiang L (2018) Selective offloading in mobile edge computing for the green Internet of Things. IEEE Netw 32(1):54-60

Mahmood Z (2011) Cloud computing: Characteristics and deployment approaches. In: IEEE 11th international conference on computer and information technology, pp 121-126

Mastelic T, Brandic I (2015) Recent trends in energy-efficient cloud computing. IEEE Cloud Comput 2(1):40-47

Mehta A, Bayuh Lakew E, Tordsson J, Elmroth E (2018) Utility-based allocation of industrial IoT applications in mobile edge clouds

Memon RA, Leung DY, Liu CH (2009) An investigation of urban heat island intensity (UHII) as an indicator of urban heating. Atmos Res 94(3):491-500

Menouar H, Guvenc I, Akkaya K, Uluagac AS, Kadri A, Tuncer A (2017) UAV-enabled intelligent transportation systems for the smart city: applications and challenges. IEEE Commun Mag 55(3):22-28

Meulen Rvd (2017) Newsroom. [Online] Accessed on 10 May 2019. https://www.gartner.com/en/ newsroom/press-releases/2017-02-07-gartner-says-8-billion-connected-things-will-be-in-usein-2017-up-31-percent-from-2016 
Mirzaei PA (2015) Recent challenges in modeling of urban heat island. Sustain Cities Soc 19:200206

Mitchell S, Villa N, Stewart-Weeks M, Lange A (2013) The Internet of everything for cities: connecting people, process, data and things to improve the livability of cities and com-munities. Cisco, San Jose

Mitton N, Papavassiliou S, Puliafito A, Trivedi KS (2012) Combining Cloud and sensors in a smart city environment

Moore RT, Hansen MC (2011) Google Earth Engine

Morabito R, Cozzolino V, Ding AY, Beijar N, Ott J (2018) Consolidate IoT edge computing with lightweight virtualization. IEEE Netw 32(1):102-111

Morán AJ, Profaizer P, Zapater MH, Valdavida MA, Bribián IZ (2016) Information and communications technologies (ICTs) for energy efficiency in buildings: review and analysis of results from EU pilot projects. Energy Build 127:128-137

Morris KI, Salleh SA, Chan A, Ooi MCG, Abakr YA, Oozeer MY, Duda M (2015) Computational study of urban heat island of Putrajaya, Malaysia. Sustain Cities Soc 19:359-372

NASA Cloud Computing Platform. https://www.nasa.gov/offices/ocio/ittalk/06-2010_cloud_comp uting.html. Accessed March 2010

NASA Earth Science Data Systems Program Highlights 2018, April 26, 2019, https://cdn.earthd ata.nasa.gov/conduit/upload/11232/ESDISHighlights.pdf

Ngoko Y, Saintherant N, Cerin C, Trystram D (2018) How future buildings could redefine distributed computing. In: 2018 IEEE international parallel and distributed processing symposium workshops (IPDPSW), pp 1232-1240

Ning Z, Kong X, Xia F, Hou W, Wang X (2019) Green and sustainable cloud of things: Ena-bling collaborative edge computing. IEEE Commun Mag 57(1):72-78

Nowicka K (2014) Smart city logistics on cloud computing model. Proc Soc Behav Sci 151:266-281

Oke TR (1982) The energetic basis of the urban heat island. Q J R Meteorol Soc 108(455):1-24

Petkova EP, Vink JK, Horton RM, Gasparrini A, Bader DA, Francis JD, Kinney PL (2016) Towards more comprehensive projections of urban heat-related mortality: estimates for New York City under multiple population, adaptation, and climate scenarios. Environ Health Perspect 125(1):4755

Qi H, Gani A (2012) Research on mobile cloud computing: review, trend and perspectives. In: 2012 second international conference on digital information and communication technology and its applications (DICTAP), pp 195-202

Raja CV, Chitra K, Jonafark M (2018) A survey on mobile cloud computing. Int J Sci Res Comput Sci Eng Inf Technol 3(3)

Rao AM, Rao KR (2012) Measuring urban traffic congestion: a review. Int J Traffic Transp Eng 2(4)

Rathore P, Rao AS, Rajasegarar S, Vanz E, Gubbi J, Palaniswami M (2018) Real-time urban microclimate analysis using internet of things. IEEE Internet of Things J 5(2):500-511

Ravanelli R, Nascetti A, Cirigliano R, Di Rico C, Leuzzi G, Monti P, Crespi M (2018) Monitoring the impact of land cover change on surface urban heat island through Google Earth Engine: proposal of a global methodology, first applications and problems. Remote Sens 10(9):1488

Ravanelli R, Nascetti A, Cirigliano RV, Di Rico C, Monti P, Crespi M (2018) Monitoring urban heat island through Google Earth Engine: Potentialities and difficulties in different cities of the United States. Int Arch Photogram Remote Sens Spatial Inf Sci 42(3)

Rittinghouse JW, Ransome JF (2016) Cloud computing: implementation, management, and security. CRC Press

Rizwan AM, Dennis LY, Chunho LIU (2008) A review on the generation, determination and mitigation of Urban Heat Island. J Environ Sci 20(1):120-128

Rong H, Zhang H, Xiao S, Li C, Hu C (2016) Optimizing energy consumption for data centers. Renew Sustain Energy Rev 58:674-691 
Rushikesh R, Sivappagari CMR (2015) Development of IoT based vehicular pollution monitoring system. In: 2015 international conference on green computing and Internet of Things (ICGCIoT), pp 779-783

Santamouris M (2015) Analyzing the heat island magnitude and characteristics in one hundred Asian and Australian cities and regions. Sci Total Environ 512:582-598

Santamouris M, Papanikolaou N, Livada I, Koronakis I, Georgakis C, Argiriou A, Assima-kopoulos DN (2001) On the impact of urban climate on the energy consumption of buildings. Sol Energy 70(3):201-216

Sarrat C, Lemonsu A, Masson V, Guedalia D (2006) Impact of urban heat island on regional atmospheric pollution. Atmos Environ 40(10):1743-1758

Satyanarayanan M (2010) Mobile computing: the next decade. In: Proceedings of the 1st ACM workshop on mobile cloud computing and services: social networks and beyond (MCS)

Schuelke-Leech BA, Barry B, Muratori M, Yurkovich BJ (2015) Big Data issues and opportunities for electric utilities. Renew Sustain Energy Rev 52:937-947

Seitz A, Johanssen JO, Bruegge B, Loftness V, Hartkopf V, Sturm M (2017) A fog architecture for decentralized decision making in smart buildings. In: Proceedings of the 2nd international workshop on science of smart city operations and platforms engineering, pp 34-39

Sen J (2015) Security and privacy issues in cloud computing. In: Cloud technology: concepts, methodologies, tools, and applications. IGI Global, pp 1585-1630

Sharma PP, Navdeti CP (2014) Securing Big Data hadoop: a review of security issues, threats and solution. Int J Comput Sci Inf Technol 5(2):2126-2131

Sheikhi A, Rayati M, Bahrami S, Ranjbar AM, Sattari S (2015) A cloud computing framework on demand side management game in smart energy hubs. Int J Electr Power Energy Syst 64:10071016

Shi W, Cao J, Zhang Q, Li Y, Xu L (2016) Edge computing: vision and challenges. IEEE Internet of Things J 3(5):637-646

Smarter Cities with Edge Computing. n.d. Smart Cities World. Accessed on 26 Mar 2019. https:// www.smartcitiesworld.net/opinions/opinions/smarter-cities-with-edge-computing-

Song M, Cen L, Zheng Z, Fisher R, Liang X, Wang Y, Huisingh D (2017) How would Big Data support societal development and environmental sustainability? Insights and practices. J Cleaner Product 142:489-500

Souza GBDC, Vieira FHT, Lima CR, Deus GADJ, De Castro MS, De Araujo SG, Vasques TL (2016) Developing smart grids based on GPRS and ZigBee technologies using queueing modeling-based optimization algorithm. Etri J 38(1):41-51

State of Green Denmark, 10 examples of smart city solution, Nov 01 2018. https://stateofgreen. com/en/partners/state-of-green/news/10-examples-of-smart-city-solutions/

Subashini S, Kavitha V (2011) A survey on security issues in service delivery models of cloud computing. J Netw Comput Appl 34(1):1-11

Sun X, Ansari N (2016) EdgeIoT: mobile edge computing for the Internet of Things. IEEE Commun Mag 54(12):22-29

Talukdar AK (2010) Mobile computing. 2nd edn. Tata McGraw-Hill Education

Tong L, Li Y, Gao W (2016) A hierarchical edge cloud architecture for mobile computing. In: IEEE INFOCOM 2016-The 35th annual IEEE international conference on computer communications, pp $1-9$

Tran TX, Pandey P, Hajisami A, Pompili D (2017) Collaborative multi-bitrate video caching and processing in mobile-edge computing networks. In: 2017 13th annual conference on wireless on-demand network systems and services (WONS), pp 165-172

Tran TX, Hajisami A, Pandey P, Pompili D (2017) Collaborative mobile edge computing in 5G networks: new paradigms, scenarios, and challenges. IEEE Commun Mag 55(4):54-61

Tsahalis J, Tsahalis HT, Moussas VC (2013) Optimization of a heterogeneous simulations workflow. In: 5th international conference on experiments/process / system modelling/simulation / optimization (5th IC-EpsMsO) 
Van Weverberg K, De Ridder K, Van Rompaey A (2008) Modeling the contribution of the Brussels heat island to a long temperature time series. J Appl Meteorol Climatol 47(4):976-990

Vlahogianni EI, Kepaptsoglou K, Tsetsos V, Karlaftis MG (2016) A real-time parking prediction system for smart cities. J Intell Transport Syst 20(2):192-204

Voogt JA, Oke TR (2003) Thermal remote sensing of urban climates. Remote Sens Environ 86(3):370-384

Wang H, Gong J, Zhuang Y, Shen H, Lach J (2017) Healthedge: Task scheduling for edge computing with health emergency and human behavior consideration in smart homes. In: 2017 IEEE international conference on Big Data, pp 1213-1222

Wang L, Jie W, Chen J (2018) Grid computing: infrastructure, service, and applications. CRC Press

Washburn D, Sindhu U, Balaouras S, Dines RA, Hayes N, Nelson LE (2009) Helping CIOs understand "smart city" initiatives. Growth 17(2):1-17

What Is Edge Computing?|GE Digital. n.d. Accessed on 26 Mar 2019. https://www.ge.com/digital/ blog/what-edge-computing

Wibowo S, Wells M (2018) Green cloud computing and economics of the cloud: moving to-wards sustainable future. GSTF J Comput (JoC) 5(1)

Wong J, Wang X, Li H, Chan G (2014) A review of cloud-based BIM technology in the construction sector. J Inf Technol Constr 19:281-291

Xing J, Dai H, Yu Z (2018) A distributed multi-level model with dynamic replacement for the storage of smart edge computing. J Syst Architect 83:1-11

Xiong J, Thenkabail PS, Gumma MK, Teluguntla P, Poehnelt J, Congalton RG, Yadav K, Thau D (2017) Automated cropland mapping of continental Africa using Google Earth Engine cloud computing. ISPRS J Photogram Remote Sens 126:225-244

Yang C, Huang Q (2013) Spatial cloud computing: a practical approach. CRC Press, Baton Rouge, FL

Yang C, Raskin R (2009) Introduction to distributed geographic information processing re-search. Int J Geogr Inf Sci 23(5):553-560

Yang C, Li W, Xie J, Zhou B (2008) Distributed geospatial information processing: sharing distributed geospatial resources to support Digital Earth. Int J Digital Earth 1(3):259-278

Yang C, Goodchild M, Huang Q, Nebert D, Raskin R, Xu Y, Bambacus M, Fay D (2011) Spatial cloud computing: how can the geospatial sciences use and help shape cloud computing? Int $\mathrm{J}$ Digital Earth 4(4):305-329

Yang X, Huang X, Liu JK (2016) Efficient handover authentication with user anonymity and untraceability for mobile cloud computing. Future Gener Comput Syst 62:190-195

Yang C, Huang Q, Li Z, Liu K, Hu F (2017a) Big Data and cloud computing: innovation opportunities and challenges. Int J Digital Earth 10(1):13-53

Yang C, Yu M, Hu F, Jiang Y, Li Y (2017b) Utilizing cloud computing to address big geospatial data challenges. Comput Environ Urban Syst 61:120-128

Yannuzzi M, Milito R, Serral-Gracià R, Montero D, Nemirovsky M (2014) Key ingredients in an IoT recipe: fog computing, cloud computing, and more fog computing. In: 2014 IEEE 19th international workshop on Computer Aided Modeling and Design of Communication Links and Networks (CAMAD), pp 325-329

Yao Y, Peng Z, Xiao B, Guan J (2017) An efficient learning-based approach to multi-objective route planning in a smart city. In: 2017 IEEE International Conference on Communications (ICC), pp $1-6$

Yi S, Qin Z, Li Q (2015) Security and privacy issues of fog computing: a survey. International conference on wireless algorithms, systems, and applications. Springer, Cham, pp 685-695

Yi S, Hao Z, Zhang Q, Zhang Q, Shi W, Li Q (2017) Lavea: latency-aware video analytics on edge computing platform. In: Proceedings of the second ACM/IEEE symposium on edge computing, p 15

Yu Z, Yao Y, Yang G, Wang X, Vejre H (2019) Spatiotemporal patterns and characteristics of remotely sensed region heat islands during the rapid urbanization (1995-2015) of Southern China. Sci Total Environ 674:242-254 
Zhang H, Ye C (2016) An indoor navigation aid for the visually impaired. In: 2016 IEEE international conference on robotics and biomimetics (ROBIO), pp 467-472

Zhang X, Zhong T, Feng X, Wang K (2009) Estimation of the relationship between vegetation patches and urban land surface temperature with remote sensing. Int J Remote Sens 30(8):21052118

Zhang Q, Cheng L, Boutaba R (2010) Cloud computing: state-of-the-art and research challenges. J Internet Serv Appl 1(1):7-18

Zheng P, Ni L (2010) Smart phone and next generation mobile computing. Elsevier

Zhong S, Qian Y, Zhao C, Leung R, Wang H, Yang B, Fan J, Yan H, Yang XQ, Liu D (2017) Urbanization-induced urban heat island and aerosol effects on climate extremes in the Yangtze River Delta region of China. Atmos Chem Phys 17(8):5439-5457

Zhou J, Cao Z, Dong X, Vasilakos AV (2017) Security and privacy for cloud-based IoT: challenges. IEEE Commun Mag 55(1):26-33
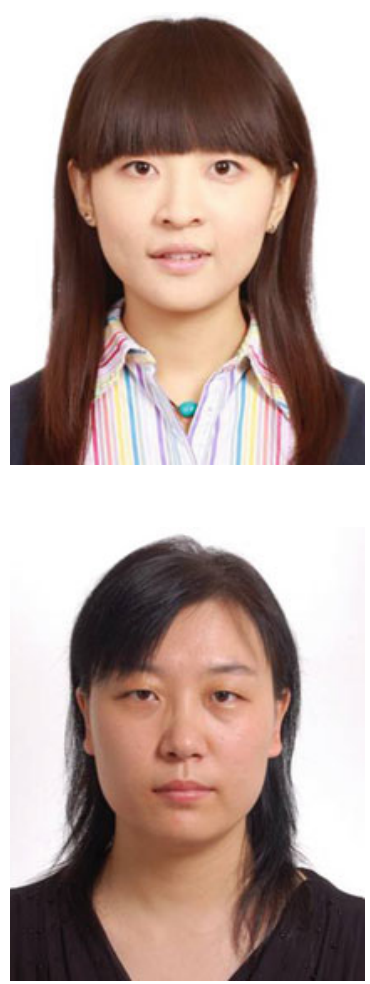

Qian Liu is a Ph.D. candidate majoring in Geography and Geoinformation Science (GGS) at George Mason University (GMU). She serves as a graduate research assistant in the National Science Foundation (NSF) Spatiotemporal Innovation Center. Her research mainly focuses on geographical events detection and segmentation, machine learning applications in natural phenomena, climate data downscaling, global precipitation climatology analysis, remote sensing, and geographical data fusion.

Juan Gu is Senior Engineer of Geographical Information Science at Beijing Institute of Surveying and Mapping. She is interested in building smart cities using cut-edge geospatial technologies. 


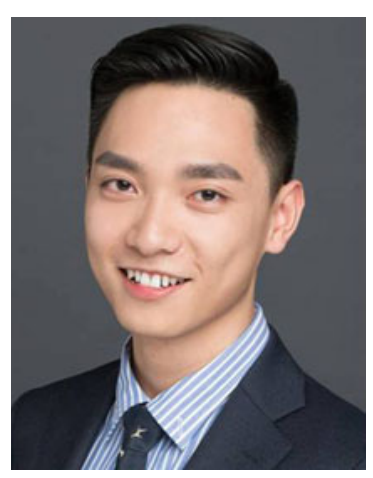

Jingchao Yang is a Ph.D. candidate majoring in Geoinformation Sciences at GMU. He has worked on several NSF and NASA funded projects as a Research Assistant for the NSF Spatiotemporal Innovation Center. He is currently applying the IoT dataset and machine learning algorithms to build a temperature forecasting model in urban areas.

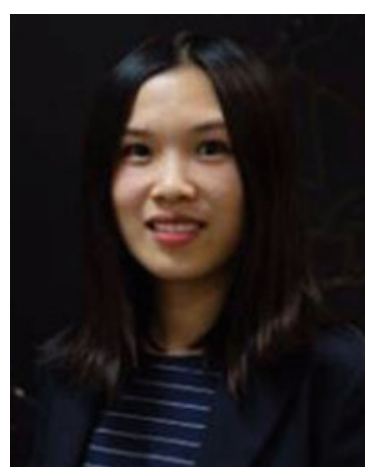

Yun Li is a Ph.D. candidate majoring in Earth Systems and Geoinformation Sciences (ESGS) at GMU. Her research mainly focuses on improving geospatial data discovery using machine learning-based methods, high-performance computing, and outreaches to spatiotemporal analytics for environmental and climate data.

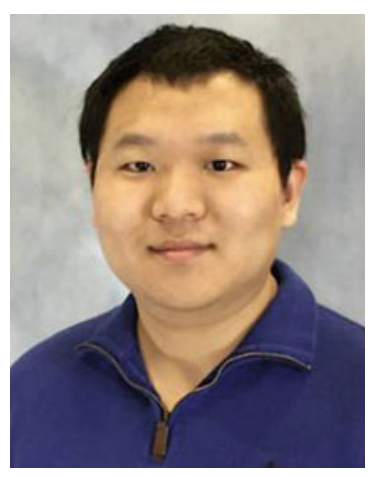

Dexuan Sha is a Ph.D. candidate majoring in ESGS at GMU. He serves as a graduate research assistant in the NSF Spatiotemporal Innovation Center. His research mainly focuses on cyberinfrastructure and big data platforms, high spatial resolution remote sensing, spatiotemporal computing, and knowledge graphing. 

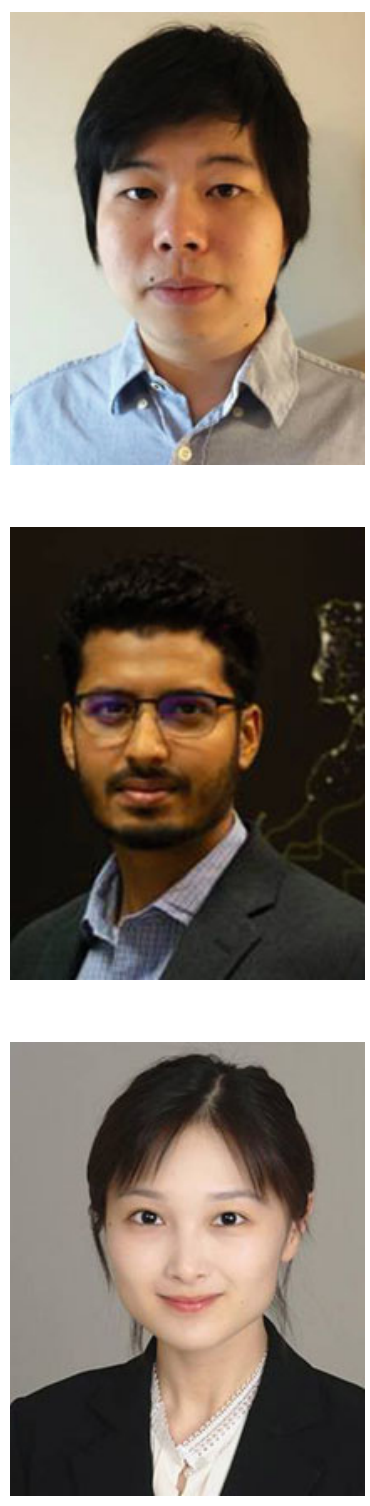

Mengchao Xu obtained his Ph.D. from the GGS Department at GMU. His research mainly focuses on cloud computing, highperformance computing networks, spatial database systems, and precipitation downscaling. He is a GIS data engineer for autonomous driving systems.

Ishan Shams is a Ph.D. student majoring in ESGS at GMU. His research mainly focuses on high-performance computing and spatiotemporal platform visualization.

Manzhu Yu is Assistant Professor of GIScience at the Department of Geography, Pennsylvania State University. She received her bachelor's degree in Remote Sensing from Wuhan University in 2012 and doctoral degree in Earth System and Geoinformation Science from George Mason University in 2017. Her research focuses on spatiotemporal theories and applications, atmospheric modeling, environmental analytics, big data and cloud computing, and the capability to use the above to solve pressing issues in natural hazards and sustainability. 


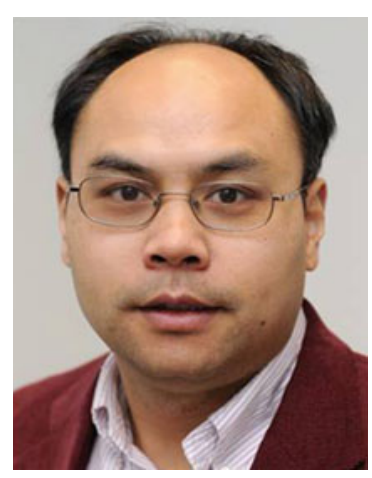

Chaowei Yang is Professor of Geographical Information Science at George Mason University, where he founded and directs the Center for Intelligent Spatial Computing and the NSF Spatiotemporal Innovation Center. He is interested in analyzing, learning, mining, and identifying spatiotemporal patterns and principles to enable scientific discovery and engineering development.

Open Access This chapter is licensed under the terms of the Creative Commons Attribution 4.0 International License (http://creativecommons.org/licenses/by/4.0/), which permits use, sharing, adaptation, distribution and reproduction in any medium or format, as long as you give appropriate credit to the original author(s) and the source, provide a link to the Creative Commons license and indicate if changes were made.

The images or other third party material in this chapter are included in the chapter's Creative Commons license, unless indicated otherwise in a credit line to the material. If material is not included in the chapter's Creative Commons license and your intended use is not permitted by statutory regulation or exceeds the permitted use, you will need to obtain permission directly from the copyright holder.

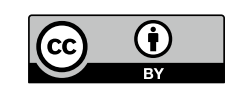

